\title{
Defect-induced condensation and central peak at structural transitions
}

\author{
F. Schwabl and U. C. Täuber \\ Institut für Theoretische Physik, Physik-Department der Technischen Universität München, \\ James-Franck-Strasse, D-8046 Garching, Germany \\ (Received 11 October 1990)
}

\begin{abstract}
We investigate how the statics and dynamics of distortive phase transitions are influenced by defects which locally increase the transition temperature. In contrast to the local condensation at a single defect, a finite concentration $n$ of randomly distributed defects induces a real phase transition at the temperature $T_{c}(n)$. The phonon response function is calculated analytically in single-site approximation. For $T>T_{c}(n)$ and low phonon damping we find a soft-phonon impurity band below the continuum, which eventually becomes overdamped when $T \rightarrow T_{c}(n)$ and produces a dynamical central peak in the phonon-phonon correlation function. In the case of stronger damping and low defect concentration, the impurity band is masked and only appears as a narrow central peak very close to $T_{c}(n)$. We develop a method which allows the computation of the order parameter and the correlation function in random systems. For $T<<T_{c}(n)$, the dynamical central peak disappears in the longitudinal correlation function, while it persists in the transverse components for rotational symmetry. The effect of cubic terms breaking the continuous rotational invariance is also investigated. In any case, the finite order parameter below $T_{c}(n)$ produces a static central peak in the dynamical structure factor, which has a finite width in momentum space.
\end{abstract}

\section{INTRODUCTION}

We study the influence of a finite concentration of randomly distributed defects, which locally increase the transition temperature, on the statics and dynamics of structural phase transitions. Although we formulate the theory in the context of distortive phase transitions, it is general enough to be applicable to other types of transitions as well.

The influence of imperfections on the static critical behavior near second-order phase transitions has been of interest for a long time. ${ }^{1,2}$ An additional stimulus to studying the influence of defects came from the observation of a central peak at $\omega=0$ in the dynamical structure factor above the transition temperature in $\mathrm{SrTiO}_{3}$ (Refs. 3 and 4) and a variety of other substances undergoing structural transitions. ${ }^{5-10}$ For a review of the experimental results, see Ref. 11. The widths of the observed central peaks are so small that they have eluded direct experimental determination ${ }^{12-16}$ in most cases; e.g., in $\mathrm{SrTiO}_{3}$ an upper limit from Mössbauer scattering is $10^{-8} \mathrm{eV} .{ }^{14}$ There are indications that the strength of the central peak depends on the purity of the sample. $9,10,17-19$ While it is obvious that any slowly relaxing mode which couples to the order parameter would give rise to a central peak, ${ }^{20}$ explicit microscopic anharmonic lattice-dynamical theories ${ }^{21-25}$ led to widths which are typically phonon linewidths.

The narrowness of the observed peaks then prompted the suggestion that they might be caused by static defects which couple linearly to the order parameter. These would give rise to elastic Huang scattering ${ }^{26-28}$ and to a critical anomaly in the inhomogeneous electron paramagnetic resonance (EPR) linewidth. It was also realized that the defects could give a dynamical central peak if they were not static, but would hop between different positions or had internal degrees of freedom which couple to the order parameter. ${ }^{29,30}$ Also, a lattice-dynamical model with defect cells was investigated and a dynamical central peak was obtained by combining mean-field theory and average crystal approximation. ${ }^{30}$ Defects which increase the transition temperature $T_{c}^{0}$ locally, the local ordering induced by the softening of a localized phonon at the local transition temperature $T_{c}^{l}$, and the dynamic consequences on the actual phase transition have been investigated in a Ginzburg-Landau theory. ${ }^{31,32}$ The local ordering in a discrete lattice model was later studied in a mean-field approximation. ${ }^{33,34}$ Moleculardynamics simulations were performed for one- and twodefect systems, showing the emergence of a dynamical central peak near the transition. ${ }^{35,36}$ A hint for the appearance of a central mode in doped $\mathrm{SrTiO}_{3}$ stems from a lattice-dynamical shell model. ${ }^{37}$

Now we return to the properties of defects which locally increase the transition temperature. From the study of single defects, the following picture emerged. Because of the local increase of the transition temperature, a characteristic soft localized phonon appears. Below the temperature $T_{c}^{l}$, where the frequency of this localized phonon mode vanishes, local ordering sets in near the impurities (because of this entirely local character, $T_{c}^{l}$ does not represent a real transition temperature). With decreasing temperature the amplitude and fraction of space occupied by this local condensate or cluster increases and its reorientation rate (hopping rate between equivalent orientations) decreases. With appropriate averaging of such single-defect properties, a dynamical central peak could be obtained. ${ }^{30,31}$

The present investigation goes far beyond such aver- 
aged single-defect studies in consistently treating a random impurity system of finite defect concentration $n$. It is, however, based on a Ginzburg-Landau approximation, the limitations of which will be discussed in Sec. VI. Instead of the localized states, we find a narrow impurity band below the usual optic-phonon continuum. This impurity band softens at $T_{c}(n)$, a concentration-dependent temperature which we call the "defect-induced transition temperature" and which constitutes a proper bulk phase transition. When approaching $T_{c}(n)$ from above, the softening impurity band eventually becomes overdamped and gives rise to a narrow dynamical central peak. Below $T_{c}(n)$, a finite order parameter is found that is concentrated at the defects, but also spreads throughout the whole sample. If the impurity concentration is low, the average order parameter will be very small in the temperature range between $T_{c}(n)$ and the bulk transition temperature $T_{c}^{0}$ of the pure system and will acquire an experimentally observable value only near and below $T_{c}^{0}$, similar to the order parameter of a pure crystal, but somewhat rounded.

Furthermore, the finite average displacement below $T_{c}(n)$ produces a static central peak in the scattering cross section, which, because of the randomly inhomogeneous static order-parameter configuration, contains a contribution with finite width in momentum space (finite " $q$ width") in addition to the Bragg part. The intensity of this central peak of zero frequency width increases when the temperature is lowered to $T_{c}^{0}$.

Below $T_{c}(n)$, the fate of the dynamical central peak stemming from the impurity band depends on the number $M$ of components of the order parameter, on the symmetry, and on the coupling coefficients of the nonlinear terms. In the longitudinal component, the central peak broadens and disappears into the continuum of the optic phonons, which continue to soften, but remain at a finite frequency even at $T_{c}^{0}$ because of the small, but finite order parameter. Hence, for a one-component order parameter and in the longitudinal direction of a multicomponent order parameter, there is no dynamical central peak further away from $T_{c}(n)$ and, in particular, near $T_{c}^{0}$. In an $M$ component system $(M>1)$, the transverse components, however, show quite an interesting behavior for $T<T_{c}(n)$. In the case of continuous rotational invariance (Heisenberg symmetry), the narrow dynamical central peak persists in the ordered phase. For weak anisotropy it remains in a rather large temperature range below $T_{c}(n)$, while for strong anisotropy the dynamical central peak acquires an increasing stiffness constant and disappears into the continuum of extended phonon states. None of the contributions to the scattering cross section diverge at $T_{c}^{0}$ because of the finite order parameter.

This paper is organized as follows: In Sec. II we present our model free energy, both in a continuum (Ginzburg-Landau) and lattice formulation, taking into account the influence of locally "softening" defects. The basic equations for the statics and dynamics of distortive structural phase transitions will be derived and the necessary correlation functions defined. Section III contains a review of earlier results (mainly from Ref. 32) on local order-parameter condensation near a single defect, dealing also with the question of how this phenomenon appears in the soft-phonon dynamics. In the following section, we generalize these results to a lattice with a finite concentration $n$ of randomly distributed defects. By taking an average over defect configurations, we are able to compute the phonon response and correlation functions analytically in a single-site approximation. Within our Ginzburg-Landau approximation, the defects induce a real phase transition at a shifted transition temperature $T_{c}(n)$. Closely above $T_{c}(n)$, a phonon impurity band becomes overdamped and produces a dynamical central peak. In Sec. $\mathrm{V}$ we perform a self-consistent calculation of the spatially inhomogeneous order parameter below $T_{c}(n)$, leading to a static central peak with finite $q$ width in the scattering cross section. We also treat certain special cases in which an additional dynamical central peak in the phonon correlation function can be found due to rotational invariance and investigate the influence of cubic terms. Finally, in Sec. VI we summarize our results and discuss their range of validity. In Appendix $A$ the scattering cross section is evaluated for more general lattices than in Sec. II B, and in Appendix B a different derivation of the phonon response function is given.

\section{GENERAL EQUATIONS}

In this section we introduce our model describing the influence of softening defects on second-order distortive (displacive) phase transitions, both in a continuum and in a discrete version. Furthermore, we define the densitydensity correlation function $S(\mathbf{k}, \omega)$ and discuss its decomposition into the elastic parts (Bragg peak, Huang scattering) and into the dynamical phonon-phonon correlation function which is related to the retarded phonon Green's function (response function).

\section{A. Model}

Following the ideas of Ginzburg-Landau theory, we expand the free energy $\mathcal{F}$ in terms of the displacement field $\psi^{\alpha}$ serving as the appropriate order parameter in the case of (ferro)distortive structural phase transitions:

$$
\begin{aligned}
\mathscr{F}\left[\left\{\psi^{\alpha}\right\}\right]=\int & {\left[\frac{1}{2} \sum_{\alpha}\left[a-\sum_{i_{D}=1}^{N_{D}} U\left(\mathbf{x}-\mathbf{x}_{i_{D}}\right)\right]\left[\psi^{\alpha}(\mathbf{x})\right]^{2}\right.} \\
& +\frac{c}{2} \sum_{\alpha}\left[\nabla \psi^{\alpha}(\mathbf{x})\right]^{2}+\frac{b-v}{4}\left[\sum_{\alpha}\left[\psi^{\alpha}(\mathbf{x})\right]^{2}\right]^{2} \\
& \left.+\frac{v}{4} \sum_{\alpha}\left[\psi^{\alpha}(\mathbf{x})\right]^{4}-h(\mathbf{x}) \psi^{\mathrm{L}}(\mathbf{x})\right] d^{d} x .
\end{aligned}
$$

Here $\alpha=1,2, \ldots, M$ denote the components of the order parameter field, with $\alpha=\mathrm{L}$ marking the longitudinal direction parallel to an external field $h$. As usual, the harmonic coefficient $a=a^{\prime}\left(T-T_{c}^{0}\right)$ depends linearly on the temperature $T$, vanishing at the bulk transition temperature $T_{c}^{0}$ of the pure system. The stiffness constant $c$ has to be positive in order that spatially uniform configurations be energetically preferred to inhomogene- 
ous ones. The coefficients of the nonlinear contributions, i.e., $b-v$ of the rotationally invariant part and $v$ of the cubic term, have to fulfill the constraint $b>0$ for stability reasons. Finally, the short-range potential $U(\mathbf{x})$ describes the influence of localized defects, distributed at the randomly selected points $\mathbf{x}_{i_{D}}, i_{D}=1,2, \ldots, N_{D}$.

In Eq. (2.1) we restrict our discussions to impurities that break the translational invariance of the harmonic Ginzburg-Landau coefficient only, neglecting any influence on the gradient and nonlinear terms. $U>0$ then corresponds to a local increase of the transition temperature, implying a softening of the material near the defects. More generally, localized defects give rise to an interaction Hamiltonian containing also a linear coupling to the order-parameter field $\propto \int \sum_{\alpha} \tau_{\lambda}^{\alpha} \psi^{\alpha}(\mathbf{x}) d^{d} x .{ }^{31}$ In the latter case, $\tau_{\lambda}^{\alpha}$ characterizes different orientations of the defect. Now two cases have to be distinguished. The first possibility is that $\tau_{\lambda}^{\alpha}$ is fast compared with $\psi^{\alpha}(\mathbf{x})$ and thus follows the order-parameter motion adiabatically. Then $\tau_{\lambda}^{\alpha}$ can be eliminated, yielding an attractive quadratic coupling to the order parameter which leads us back to Eq. (2.1). The second possibility is that the defect reorientation is slow compared with the order parameter, in which situation we have to distinguish further between two cases. If the reorientation time is so slow that the defects can be considered as static, the result is a static deformation of the lattice with a range of the order of the correlation length, giving rise to a static central peak of vanishing width and intensity proportional to the square of the static response function (Huang scattering) and also proportional to the defect concentration for statistically independent defects. ${ }^{26-28}$ An additional dynamical central peak stems from the broken symmetry in the defect neighborhood and the ensuing coupling of the order parameter to heat diffusion. ${ }^{31}$ If the relaxation time is finite, but the relaxation still slow, the defects provide a slowly relaxing mode. This leads exactly to the orderparameter susceptibility used for phenomenological descriptions of the observed central peaks. ${ }^{20,30}$

After this digression we return to Eq. (2.1). In the framework of Ginzburg-Landau theory, the static equilibrium states can be found by solving the stationarity condition

$$
\left.\frac{\delta \mathscr{F}\left[\left\{\psi^{\alpha}\right\}\right]}{\delta \psi^{\alpha}(\mathbf{x})}\right|_{\psi^{\alpha}=\bar{\psi}^{\alpha}}=0,
$$

with the constraint $\mathscr{F}[\bar{\psi}]<\infty$ (see Sec. III A).

Furthermore, the dynamics of our model are given by the following Langevin equation for the time-dependent displacement field: ${ }^{32}$

$I\left(\frac{\partial^{2}}{\partial t^{2}}+\gamma \frac{\partial}{\partial t}\right) \psi^{\alpha}(\mathbf{x}, t)=-\frac{\delta \mathscr{F}\left[\left\{\psi^{\alpha}\right\}\right]}{\delta \psi^{\alpha}(\mathbf{x}, t)}+r^{\alpha}(\mathbf{x}, t)$,

where a mass $I$ and a phenomenological damping constant $\gamma$ for a nonconserved order parameter have been introduced. In the case of the 105-K transition of $\mathrm{SrTiO}_{3}, I$ equals half the moment of inertia of the oxygen octahedra. The probability distribution for the stochastic forces, $r^{\alpha}(\mathbf{x}, t)$, is assumed to be Gaussian with the moments

$$
\begin{aligned}
& \left\langle r^{\alpha}(\mathbf{x}, t)\right\rangle=0, \\
& \left\langle r^{\alpha}(\mathbf{x}, t) r^{\beta}\left(\mathbf{x}^{\prime}, t^{\prime}\right)\right\rangle=2 I \gamma k_{B} T \delta^{\alpha \beta} \delta\left(\mathbf{x}-\mathbf{x}^{\prime}\right) \delta\left(t-t^{\prime}\right),
\end{aligned}
$$

the latter being related to the damping constant by the usual Einstein relation. Linearizing the deterministic part of the right-hand side of Eq. (2.3a) and performing the Fourier transformation into the $(\mathbf{q}, \omega)$ space yields an eigenvalue problem, the eigenvalues being connected with the frequencies of the optic phonons (see Sec. III B).

However, a discrete version of Eq. (2.1) with a pointlike defect potential on a lattice with $N$ points turns out to be more convenient for the investigation of a system with a finite concentration of defects; i.e., $n=N_{D} / N$ remains fixed in the thermodynamic limit where $N \rightarrow \infty$. Introducing a positive defect strength $\lambda$, we have

$$
\begin{aligned}
F\left(\left\{\psi_{i}^{\alpha}\right\}\right)= & \frac{1}{2} \sum_{i, j} \sum_{\alpha, \beta} \psi_{i}^{\alpha} G_{0}^{\alpha \beta}{ }_{i j}-1 \psi_{j}^{\beta}-\frac{\lambda}{2} \sum_{i, i_{D}} \delta_{i i_{D}} \sum_{\alpha}\left(\psi_{i}^{\alpha}\right)^{2} \\
& +\frac{b-v}{4} \sum_{i}\left[\sum_{\alpha}\left(\psi_{i}^{\alpha}\right)^{2}\right)^{2} \\
& +\frac{v}{4} \sum_{i}\left(\psi_{i}^{\alpha}\right)^{4}-\sum_{i} h_{i} \psi_{i}^{\mathrm{L}}
\end{aligned}
$$

where the "free" static propagator

$$
G_{0}{ }^{\alpha \beta}{ }_{i j}=\frac{1}{N} \sum_{\mathbf{q}} e^{i \mathbf{q} \cdot\left(\mathbf{a}_{i}-\mathbf{a}_{j}\right)} G_{0}^{\alpha \beta}(\mathbf{q}),
$$

with lattice vectors $\mathbf{a}_{i}$ according to Eq. (2.1), is diagonal with respect to the order-parameter components and in momentum space reads

$$
G_{0}^{\alpha \beta-1}(\mathbf{q})=G_{0}^{-1}(q) \delta^{\alpha \beta}=\left(a+c q^{2}\right) \delta^{\alpha \beta} .
$$

In order to find the stationary solutions and to specify the dynamics of the lattice [see Eqs. (4.1) and (4.2a)], we have to take partial derivatives of $F$ with respect to the components of the displacement field at site $i$, yielding, for the longitudinal (L) and transverse $(\alpha \neq \mathbf{L})$ components,

$$
\begin{aligned}
\frac{\partial F}{\partial \psi_{i}^{\mathrm{L}}}= & \sum_{j} G_{0}{ }^{-1}{ }_{i j} \psi_{j}^{\mathrm{L}}-\lambda \sum_{i_{D}} \delta_{i i_{D}} \psi_{i}^{\mathrm{L}} \\
& +(b-v) \psi_{i}^{\mathrm{L}} \sum_{\alpha(\neq \mathbf{L})}\left(\psi_{i}^{\alpha}\right)^{2}+b\left(\psi_{i}^{\mathrm{L}}\right)^{3}-h_{i}
\end{aligned}
$$

and

$$
\begin{aligned}
\frac{\partial F}{\partial \psi_{i}^{\alpha}}= & \sum_{j} G_{0}^{-1}{ }_{i j} \psi_{i}^{\alpha}-\lambda \sum_{i_{D}} \delta_{i i_{D}} \psi_{i}^{\alpha}+(b-v)\left(\psi_{i}^{\mathrm{L}}\right)^{2} \psi_{i}^{\alpha} \\
& +(b-v) \psi_{i}^{\alpha} \sum_{\beta(\neq \alpha, \mathrm{L})}\left(\psi_{i}^{\beta}\right)^{2}+b\left(\psi_{i}^{\alpha}\right)^{3}
\end{aligned}
$$

respectively. Sections IV and V will be based on Eqs. (2.5).

The present theory comprises ferrodistortive as well as antiferrodistortive phase transitions, accompanied by 
zone-center and zone-boundary soft modes, respectively. For the former the order-parameter components $\psi_{i}^{\alpha}$ are identical with the displacements $\phi_{i}^{\alpha}$; for the latter they differ by a sign. Generally, we write

$$
\phi_{i}^{\alpha}=e^{i \mathrm{q}_{0} \cdot \mathbf{a}_{i}} \psi_{i}^{\alpha},
$$

where $\mathbf{a}_{i}$ is the lattice vector of site $i$. For a ferrodistortive transition, we have $\mathbf{q}_{0}=0$, while for an antiferrodistortive transition $\mathbf{q}_{0} \neq 0, e^{2 i \mathbf{q}_{0} \cdot \mathbf{a}_{i}}=1$. For instance, at the antiferrodistortive $105-\mathrm{K}$ transition of $\mathrm{SrTiO}_{3}$, $\mathrm{q}_{0}=\left(\pi / a_{0}, \pi / a_{0}, \pi / a_{0}\right)$, where $a_{0}$ is the lattice constant of the simple-cubic perovskite lattice.

We remark that sometimes we shall consider a onedimensional model, which does not exhibit a phase transition. Yet there is a temperature below which the most probable state acquires a finite value (fluctuations still yield a zero order parameter even then). This onedimensional most probable state serves as a model for the most probable state in three dimensions. Furthermore, in the spirit of Ginzburg-Landau theory, one approximately determines therefrom the order parameter. Once again we emphasize that we use this one-dimensional model only because of analytical simplicity. All general formulas (impurity-averaged susceptibility, order parameter, scattering intensity) will be derived in arbitrary dimension. The figures generally show the three-dimensional results. The Ginzburg-Landau parameters used for the figures (if not specified otherwise in the figure captions) are displayed in Table I. They are oriented at the values
TABLE I. Ginzburg-Landau parameters used in the graphs (if not specified in the figure captions).

$$
\begin{aligned}
& T_{c}^{0}=105 \mathrm{~K} \\
& a^{\prime}=5.4 \times 10^{-3} \mathrm{eV} \mathrm{K}^{-1} \\
& b=2.6 \times 10^{2} \mathrm{eV} \\
& c=3.7 \times 10^{-15} \mathrm{eV} \mathrm{cm}^{2} \\
& \frac{N}{V}=\frac{1}{a_{0}^{3}}=1.7 \times 10^{22} \mathrm{~cm}^{-3} \\
& \Lambda=\frac{\pi}{a_{0}}=8.1 \times 10^{7} \mathrm{~cm}^{-1} \\
& q=2.8 \times 10^{6} \mathrm{~cm}^{-1} \\
& I=1.3 \times 10^{-26} \mathrm{eV} \mathrm{s}^{2} \\
& \hbar \gamma=1.5 \times 10^{-3} \mathrm{eV}^{-5} \\
& n=\frac{N_{D}}{N}=1.5 \times 10^{-5} \\
& t_{c}(n)=\left[T_{c}(n)-T_{c}^{0}\right] / T_{c}^{0}=0.6 \\
& \quad \rightarrow \lambda=18 \mathrm{eV} \\
& U_{0}=a_{0}^{3} \lambda=1.1 \times 10^{-21} \mathrm{eV} \mathrm{cm}
\end{aligned}
$$

of $\mathrm{SrTiO}_{3}$, not taking into account, however, any anisotropies. For the defect properties (strength and concentration), arbitrary but reasonable values have been chosen.

\section{B. Density-density correlation function}

The dynamical structure factor observed in scattering experiments is related to the Fourier-transformed density-density correlation function. Denoting the thermodynamical average by $\langle\cdots\rangle$, its definition is

$$
S(\mathbf{k}, \omega)=\int e^{i \omega t}\left\langle\frac{1}{N} \sum_{1 \leq i, j \leq N} e^{-i \mathbf{k} \cdot\left[\mathbf{a}_{i}+\phi_{i}(t)\right]} e^{i \mathbf{k} \cdot\left[\mathbf{a}_{j}+\phi_{j}(0)\right]}\right\rangle d t
$$

Here $\mathbf{a}_{i}$ is a lattice vector and $\phi_{i}$ the displacement (2.6). In a system with quenched defects, $S(\mathbf{k}, \omega)$ has to be averaged over the defect configuration. This configurational average is denoted by $\langle\langle\cdots\rangle\rangle$, and its mathematical definition will be given in Sec. IV [Eq. (4.12)]. In the computation of $\langle\langle S(\mathbf{k}, \omega)\rangle\rangle$ we introduce a cumulant expansion for the combined thermal and configurational averages

$$
\begin{array}{r}
\left\langle\langle S(\mathbf{k}, \omega)\rangle=\int e^{i \omega t} \frac{1}{N} \sum_{1 \leq i, j \leq N} e^{-i \mathbf{k} \cdot\left(\mathbf{a}_{i}-\mathbf{a}_{j}\right)} \exp \left[i \mathbf{k} \cdot\left[\left\langle\left\langle\phi_{i}\right\rangle\right\rangle-\left\langle\left\langle\left\langle\phi_{j}\right\rangle\right\rangle\right\rangle\right]\right]\right. \\
\times \exp \left[-\frac{1}{2} \sum_{\alpha, \beta} k^{\alpha} k^{\beta}\left[\left\langle\left\langle\left\langle\left(\phi_{i}^{\alpha}-\phi_{j}^{\alpha}\right)\left(\phi_{i}^{\beta}-\phi_{j}^{\beta}\right)\right\rangle\right\rangle\right\rangle\right.\right. \\
\left.-\left(\left\langle\left\langle\phi_{i}^{\alpha}\right\rangle\right\rangle\right\rangle-\left\langle\left\langle\left\langle\phi_{j}^{\alpha}\right\rangle\right\rangle\right)\left(\left\langle\left\langle\left\langle\phi_{i}^{\beta}\right\rangle\right\rangle\right\rangle-\left\langle\left\langle\left\langle\phi_{j}^{\beta}\right\rangle\right\rangle\right)\right]+\cdots\right] d t .
\end{array}
$$

Next, we introduce the static local order parameter

$$
\bar{\psi}_{i}^{\alpha}=\delta^{\alpha \mathrm{L}} \bar{\psi}_{i}^{\mathrm{L}}
$$

[see Eq. (2.6)] and the dynamical fluctuations $u_{i}^{\alpha}(t)$ with vanishing thermal average $\left\langle u_{i}^{\alpha}(t)\right\rangle=0$ :

$$
\psi_{i}^{\alpha}(t)=\bar{\psi}_{i}^{\alpha}+u_{i}^{\alpha}(t)
$$

The mean order parameter $\left\langle\left\langle\bar{\psi}^{\alpha}\right\rangle\right\rangle=\left\langle\left\langle\bar{\psi}_{i}^{\alpha}\right\rangle\right\rangle$ is then independent of $i$ [see Eq. (5.3)], and the defect-averaged scattering cross section reads 


$$
\begin{aligned}
\langle\langle S(\mathbf{k}, \omega)\rangle\rangle=\int e^{i \omega t} \frac{1}{N} \sum_{1 \leq i, j \leq N} & e^{-i \mathbf{k} \cdot\left(\mathbf{a}_{i}-\mathbf{a}_{j}\right)} e^{-2 W} \\
& \times\left[\cos ^{2}(\mathbf{k} \cdot\langle\langle\bar{\psi}\rangle\rangle)+\sin ^{2}(\mathbf{k} \cdot\langle\langle\bar{\psi}\rangle\rangle) e^{i \mathbf{q}_{0} \cdot\left(\mathbf{a}_{i}-\mathbf{a}_{j}\right)}-\frac{i}{2} \sin (2 \mathbf{k} \cdot\langle\langle\bar{\psi}\rangle\rangle)\left(e^{i \mathbf{q}_{0} \cdot \mathbf{a}_{i}}-e^{-i \mathbf{q}_{0} \cdot \mathbf{a}_{j}}\right)\right] \\
& \times \exp \sum_{\alpha, \beta}\left[k^{\alpha} k^{\beta}\left(\left\langle\left\langle\bar{\psi}_{i}^{\alpha} \bar{\psi}_{j}^{\beta}\right\rangle\right\rangle-\left\langle\left\langle\bar{\psi}^{\alpha}\right\rangle\right\rangle\left\langle\left\langle\bar{\psi}^{\beta}\right\rangle\right\rangle+\left\langle\left\langle\left\langle u_{i}^{\alpha}(t) u_{j}^{\beta}(0)\right\rangle\right\rangle\right) e^{i \mathbf{q}_{0} \cdot\left(\mathbf{a}_{i}-\mathbf{a}_{j}\right)}+\cdots\right] d t\right.
\end{aligned}
$$

where $e^{2 i \mathbf{q}_{0} \cdot \mathbf{a}_{i}}=1$ has been used and the Debye-Waller factor is given by

$$
W=\frac{1}{2} \sum_{\alpha, \beta} k^{\alpha} k^{\beta}\left[\left\langle\left\langle\left(\bar{\psi}_{i}^{\alpha}-\left\langle\left\langle\bar{\psi}^{\alpha}\right\rangle\right\rangle\right)\left(\bar{\psi}_{i}^{\beta}-\left\langle\left\langle\bar{\psi}^{\beta}\right\rangle\right\rangle\right)\right\rangle\right\rangle+\left\langle\left\langle\left\langle u_{i}^{\alpha} u_{i}^{\beta}\right\rangle\right\rangle\right] .\right.
$$

Finally, we may expand the last exponential in Eq. (2.9b), with the result

$$
\begin{aligned}
\langle\langle S(\mathbf{k}, \omega)\rangle\rangle=2 \pi & N \sum_{\mathbf{g}} \delta_{\mathbf{k}, \mathbf{g}} \cos ^{2}(\mathbf{k} \cdot\langle\langle\bar{\psi}\rangle\rangle)+N \sum_{\mathbf{g}} \delta_{\mathbf{k}-\mathbf{q}_{0}, \mathbf{g}} \sin ^{2}(\mathbf{k} \cdot\langle\langle\bar{\psi}\rangle\rangle) \\
& +\sum_{\alpha, \beta} k^{\alpha} k^{\beta}\left\langle\left\langle S_{C}^{\alpha \beta}(\mathbf{k}\rangle\right\rangle \sin ^{2}(\mathbf{k} \cdot\langle\langle\bar{\psi}\rangle\rangle)+\sum_{\alpha, \beta} k^{\alpha} k^{\beta}\left\langle\left\langle S_{C}^{\alpha \beta}\left(\mathbf{k}-\mathbf{q}_{0}\right)\right\rangle\right\rangle \cos ^{2}(\mathbf{k} \cdot\langle\langle\bar{\psi}\rangle\rangle)\right] e^{-2 W} \delta(\omega) \\
& +\left(\sum_{\alpha, \beta} k^{\alpha} k^{\beta} D^{\alpha \beta}(\mathbf{k}, \omega) \sin ^{2}(\mathbf{k} \cdot\langle\langle\bar{\psi}\rangle\rangle)+\sum_{\alpha, \beta} k^{\alpha} k^{\beta} D^{\alpha \beta}\left(\mathbf{k}-\mathbf{q}_{0}, \omega\right) \cos ^{2}(\mathbf{k} \cdot\langle\langle\bar{\psi}\rangle\rangle)\right] e^{-2 W},
\end{aligned}
$$

where $\mathbf{g}$ denotes a reciprocal-lattice vector. The elastic scattering consists of four terms: (i) The first term is the standard Bragg contribution. (ii) The second term constitutes the additional Bragg peaks at $\mathbf{q}_{0}+\mathbf{g}$ due to the order parameter. [For $\mathrm{q}_{0}=\mathbf{0}$ the two form factors of (i) and (ii) add up to one.] (iii) The third and fourth terms result from random static variations of the local order parameter and can be interpreted as Huang scattering. Their intensity is determined by

$$
S_{C}^{\alpha \beta}(\mathbf{q})=\frac{1}{N} \sum_{1 \leq i, j \leq N} e^{-i \mathbf{q} \cdot\left(\mathbf{a}_{i}-\mathbf{a}_{j}\right)}\left(\bar{\psi}_{i}^{\alpha} \bar{\psi}_{j}^{\beta}-\left\langle\left\langle\bar{\psi}^{\alpha}\right\rangle\right\rangle\left\langle\left\langle\bar{\psi}^{\beta}\right\rangle\right\rangle\right)
$$

The inelastic part $D^{\alpha \beta}(\mathbf{q}, \omega)$ can be identified with the averaged dynamical phonon-phonon correlation function

$$
D^{\alpha \beta}(\mathbf{q}, \omega)=\int e^{i \omega t}\left\langle\left\langle\frac{1}{N} \sum_{1 \leq i, j \leq N} e^{-i \mathbf{q} \cdot\left(\mathbf{a}_{i}-\mathbf{a}_{j}\right)}\left\langle u_{i}^{\alpha}(t) u_{j}^{\beta}(0)\right\rangle\right\rangle\right\rangle d t
$$

which in the classical limit can be derived from the averaged phonon response function $G^{\alpha \beta}(\mathbf{q}, \omega)$ via

$$
D^{\alpha \beta}(\mathbf{q}, \omega)=\frac{2 k_{B} T}{\omega} \operatorname{Im} G^{\alpha \beta}(\mathbf{q}, \omega) .
$$

The configurational average of $(2.12)$ and the phonon response function, and herewith the inelastic scattering cross section, will be evaluated in Secs. IV and V. In Eq. (2.11), for the sake of simplicity, the polarization vectors of the order-parameter fluctuations $\psi^{\alpha}$ have been assumed to be parallel to the Cartesian axes. The generalization to lattices with a basis and to arbitrary, realistic polarization vectors is deferred to Appendix A.

\section{LOCAL CONDENSATION AT A SINGLE DEFECT}

In this section we review a number of single-defect properties from previous work, ${ }^{32}$ especially concerning the phenomenon of local order-parameter condensation. Although the treatment of the random system in the subsequent sections does not rest on these considerations, they facilitate the interpretation of the results of Secs. IV and V. It is also important to realize the significant physical and conceptional differences of the random multidefect system as compared to the single-defect properties.

Hence we consider a one-component order parameter and a single defect that locally increases the transition temperature, situated in a $d$-dimensional continuum in zero external field. Putting $h=0, M=1$, and $N_{D}=1$ (and $\mathbf{x}_{D}=\mathbf{0}$ for simplicity), Eq. (2.1) for the free energy reduces to

$$
\begin{aligned}
\mathscr{F}[\psi]=\int & {\left[\frac{1}{2}[a-U(\mathbf{x})][\psi(\mathbf{x})]^{2}\right.} \\
& \left.+\frac{c}{2}[\nabla \psi(\mathbf{x})]^{2}+\frac{b}{4}[\psi(\mathbf{x})]^{4}\right] d^{d} x,
\end{aligned}
$$

remembering $a=a^{\prime}\left(T-T_{c}^{0}\right), b>0$, and $c>0$. The probability distribution $\mathcal{P}[\psi]$ for a configuration $\psi(\mathbf{x})$ then is given by

$$
\mathscr{P}[\psi] \mathscr{D} \psi=\frac{e^{-\beta \mathscr{F}[\psi]} \mathcal{D}[\psi]}{\int e^{-\beta \mathscr{F}[\psi]} \mathcal{D}[\psi]}, \quad \beta=\frac{1}{k_{B} T} .
$$




\section{A. Statics}

In view of Eq. (3.2), the most probable states are given by the minima of the free-energy functional (3.1). The stationarity condition (2.2) now reads

$$
\left.\frac{\delta \mathscr{F}[\psi]}{\delta \psi}\right|_{\psi=\bar{\psi}}=0
$$

leading to the following nonlinear differential equation of second order:

$$
c \nabla^{2} \bar{\psi}(\mathbf{x})=[a-U(\mathbf{x})] \bar{\psi}(\mathbf{x})+b[\bar{\psi}(\mathbf{x})]^{3} .
$$

A spatially constant solution of Eq. (3.3b) is, of course, provided by $\bar{\psi}_{0}=0$, the order-parameter value of the pure system for $T>T_{c}^{0}(a>0)$. (In contrast, the constant solutions for $T<T_{c}^{0}, \bar{\psi}_{ \pm}= \pm[(-a) / b]^{1 / 2}$ describing the two possible orientations of the ordered state, are no longer allowed.) One has to be aware, however, that because of the defect influence, this homogeneous state may become unstable at a certain temperature $T_{c}^{l}$, and instead a localized order-parameter condensate (cluster) near the defect may form. ${ }^{32}$ Then $T_{c}^{l}$ is called the local transition temperature, although it does not define a proper phase transition, for the order parameter differs from zero only locally. In three dimensions the phenomenon of local condensation occurs if the defect potential $U(\mathbf{x})$ is sufficiently attractive (see Sec. IV B) and Eq. (3.3b) can be solved numerically. ${ }^{38,39}$ On approaching $T_{c}^{0}$ the localized cluster can be regarded as consisting of a core part depending on the details of the defect potential and of the following asymptotic tails:

$$
\bar{\psi}_{C}(\mathbf{x}) \propto \frac{1}{|\mathbf{x}|} e^{-|\mathbf{x}| / \xi^{>}} .
$$

Its linear dimension is given by the bulk correlation length $\xi^{>}=\sqrt{c / a}$ diverging at $T_{c}^{0}$, and also its amplitude increases, when the temperature is lowered. ${ }^{31}$

For the idealized short-range defect potential

$$
U(x)=U_{0} \delta(x), \quad U_{0}=a_{0}^{d} \lambda>0
$$

$\left[a_{0}=(V / N)^{1 / d}\right.$ is the lattice constant $]$ and just one space dimension, $d=1$, all the calculations can be performed analytically. Linear stability analysis (see Ref. 32 and Sec. III B) shows that independent of the defect strength $U_{0}$, local condensation occurs at

$$
T_{c}^{l}=T_{c}^{0}+\frac{U_{0}^{2}}{4 a^{\prime} c}
$$

For $T<T_{c}^{l}$ one finds the following stable (see Ref. 32) cluster configurations in the interval $T_{c}^{0} \leq T \leq T_{c}^{l}(a>0)$ :

$$
\bar{\psi}_{>}(x)= \pm\left(\frac{2 a}{b}\right)^{1 / 2} \frac{1}{\sinh \left(|x| / \xi^{>}+\rho^{>}\right)},
$$

where

$$
\xi^{>}=\left(\frac{c}{a}\right)^{1 / 2}, \quad \rho^{>}=\operatorname{arccoth} \frac{U_{0}}{2 \sqrt{a c}},
$$

and in the temperature range $T<T_{c}^{0}$,

$$
\bar{\psi}_{<}(x)= \pm\left(\frac{-a}{b}\right)^{1 / 2} \operatorname{coth}\left(\frac{|x|}{\xi^{<}}+\rho^{<}\right),
$$

with

$$
\xi^{<}=\left(\frac{2 c}{-a}\right)^{1 / 2}, \quad \rho^{<}=\frac{1}{2} \operatorname{arcsinh} \frac{\sqrt{-8 a c}}{U_{0}} .
$$

Note that the widths of both configurations are given by the correlation lengths $\xi^{>}$and $\xi^{<}$, respectively, of the pure system, therefore diverging with the mean-field critical exponent $\nu=\frac{1}{2}$ for $T \rightarrow T_{c}^{0}$.

In Fig. 1 we sketch the order-parameter profiles in the relevant temperature ranges. While for $T>T_{c}^{l}$ the order parameter vanishes throughout the crystal, it reaches finite values in the defect surroundings for $T<T_{c}^{l}$, induced by the local softening of the harmonic GinzburgLandau coefficient, tending to zero, however, for $|x| \rightarrow \infty$ [compare Eq. (3.7)]. Hence the condensation of the order parameter at the defect is a local phenomenon and must not be identified as a proper phase transition. A real second-order phase transition occurs at the bulk transition temperature $T_{c}^{0}$. In the ordered phase there still is a local enhancement of the order parameter near the defect, becoming less and less important for $T \ll T_{c}^{0}[\mathrm{Eq}$. (3.8)].

\section{B. Dynamics}

In this subsection we shall show how the formation of a localized cluster near a defect makes itself felt in the spectrum of the soft-phonon modes. To this end we introduce the dynamic fluctuations $u(\mathbf{x}, t)$ around the static order parameter $\bar{\psi}(\mathbf{x})$ into Eq. (2.3a), which, for vanishing damping $\gamma=0$, reduces to

$$
I \ddot{u}(\mathbf{x}, t)=-\left.\frac{\delta \mathscr{F}[\psi]}{\delta \psi(\mathbf{x}, t)}\right|_{\psi=\bar{\psi}+u}
$$

Now we neglect all terms higher than first order in $u(\mathbf{x}, t)$ and introduce the ansatz $u(\mathbf{x}, t)=\sum_{\lambda} c_{\lambda} u_{\lambda}(\mathbf{x}) e^{-i \omega_{\lambda} t}$, which leads to the following eigenvalue problem:

$I \omega_{\lambda}^{2} u_{\lambda}(\mathbf{x})=\left\{a-U(\mathbf{x})+3 b[\bar{\psi}(\mathbf{x})]^{2}-c \nabla^{2}\right\} u_{\lambda}(\mathbf{x})$.

Above $T_{c}^{l}$ the order parameter vanishes in the most probable state, $\bar{\psi}(\mathbf{x})=0$, and the eigenstates are scattering

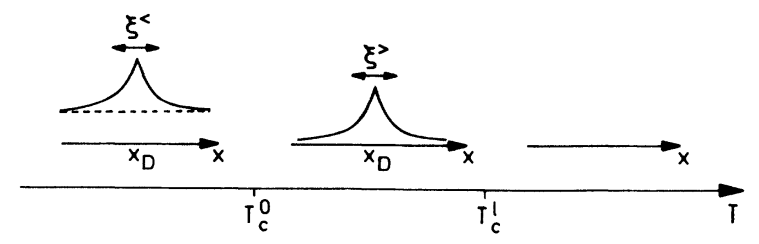

FIG. 1. Sketch of the order-parameter profiles in a singledefect system in the temperature ranges $T<T_{c}^{0}, T_{c}^{0} \leq T \leq T_{c}^{l}$, and $T>T_{c}^{l}$, respectively. 
states (certain linear combinations of $e^{i \mathbf{q} \cdot \mathbf{x}}$ and $e^{-i \mathbf{q} \cdot \mathbf{x}}$ ) with the eigenvalues

$$
\omega_{q}^{2}=\frac{a+c q^{2}}{I}
$$

i.e., propagating optic-phonon modes, which soften at the bulk transition temperature $T_{c}^{0}(a=0)$ for $q=0$. Furthermore, for sufficiently attractive defect potential $U(\mathbf{x})$ [see the discussion preceding Eq. (3.4)], there exists at least one localized phonon state $u_{l}(\mathbf{x})$ whose eigenvalue $\omega_{l}^{2}$ lies below this bulk phonon continuum. When the temperature is lowered, its eigenfrequency decreases and finally vanishes at $T=T_{c}^{l}$, corresponding to the formation of the order-parameter cluster. The bound state then "freezes in" and forms the localized condensate. The occurrence of a localized mode in the soft-phonon spectrum thus can be interpreted as a precursor to the local condensation.

In the simple one-dimensional case with defect $\delta$ potential (3.5), an explicit calculation yields, for the localized phonon mode above $T_{c}^{l}$,

$$
u_{l}(x)=\sqrt{\kappa} e^{-\kappa|x|}, \quad \kappa=\frac{U_{0}}{2 c} .
$$

Its eigenfrequency lies in the gap below the continuum

$$
\omega_{l}^{2}=\frac{1}{I}\left[a-\frac{U_{0}^{2}}{4 c}\right)=\frac{a^{\prime}}{I}\left(T-T_{c}^{l}\right),
$$

and the localized phonon mode becomes soft when the local condensation at the defect occurs, corresponding to the fact that the constant solution $\bar{\psi}_{0}=0$ becomes unstable at $T_{c}^{l}$. Below $T_{c}^{l}$ the eigenstates and eigenvalues of Eq. (3.10) have also been calculated in Ref. 32. Just below $T_{c}^{l}$ a localized mode remains in addition to the extended states, which, however, stiffens because of the buildup of the local condensate and merges into the continuum for $T \rightarrow T_{c}^{0}+3 U_{0}^{2} / 20 a^{\prime} c$.

\section{CONDENSATION FOR A FINITE DEFECT CONCENTRATION}

Of course, the problem of not just a single, but a large number $N_{D}$ of defects, their concentration $n=N_{D} / N$ remaining finite in the thermodynamic limit $N \rightarrow \infty$, is by far more promising to provide an acceptable picture for the properties of impurity-doped crystals. We emphasize that we consider quenched defects sitting on fixed, randomly distributed positions. Hence one has to perform both thermodynamic and configurational averages; i.e., after computing the thermodynamic quantities for a fixed configuration, these have to be averaged over the random positions of the defects. Of course, an exact solution for a random defect distribution, comparable to the singledefect problem, is impossible; nevertheless, the leading concentration dependence can be determined. In the first part of this section, we shall describe a systematic perturbation expansion for the propagator and its diagrammatic representation, while the second part is devoted to the discussion of the resulting response and correlation func- tions and the physics behind the emerging dynamical central peak.

\section{A. Perturbation theory and diagrammatics}

It is now more convenient to use the discrete version of our model because of the inevitable configurational average over defects at sites $i_{D}=1,2, \ldots, N_{D}$. According to Ginzburg-Landau theory, the most probable state, later to be identified with the static order parameter, is given by the solution of [compare Eq. (2.2)]

$$
\left.\frac{\partial F\left(\left\{\psi_{i}^{\alpha}\right\}\right)}{\partial \psi_{i}^{\alpha}}\right|_{\psi_{i}^{\alpha}=\bar{\psi}_{i}^{\alpha}}=0 \text {. }
$$

Equation (2.3a) for the fluctuations about this most probable configuration becomes

$$
I\left[\frac{\partial^{2}}{\partial t^{2}}+\gamma \frac{\partial}{\partial t}\right] u_{i}^{\alpha}(t)=-\left.\frac{\partial F\left(\left\{\psi_{i}^{\alpha}\right\}\right)}{\partial \psi_{i}^{\alpha}(t)}\right|_{\psi_{i}^{\alpha}=\bar{\psi}_{i}^{\alpha}+u_{i}^{\alpha}}+r_{i}^{\alpha}(t),
$$

the Gaussian stochastic forces obeying

$$
\begin{aligned}
& \left\langle r_{i}^{\alpha}(t)\right\rangle=0, \\
& \left\langle r_{i}^{\alpha}(t) r_{j}^{\beta}\left(t^{\prime}\right)\right\rangle=2 I \gamma k_{B} T \delta^{\alpha \beta} \delta_{i j} \delta\left(t-t^{\prime}\right),
\end{aligned}
$$

an Einstein relation analogous to Eq. (2.3c).

The equation of motion (4.2a) can be simplified further by linearization, using (4.1):

$$
\begin{aligned}
I\left[\frac{\partial^{2}}{\partial t^{2}}\right. & \left.+\gamma \frac{\partial}{\partial t}\right] u_{i}^{\alpha}(t) \\
& =-\left.\sum_{j} \sum_{\beta} \frac{\partial^{2} F\left(\left\{\psi_{i}^{\alpha}\right\}\right)}{\partial \psi_{i}^{\alpha} \partial \psi_{j}^{\beta}}\right|_{\psi_{i}^{\alpha}=\bar{\psi}_{i}^{\alpha}} u_{j}^{\beta}+r_{i}^{\alpha}(t) .
\end{aligned}
$$

To start out we study the situation $h=0$ and $\bar{\psi}_{i}^{\alpha}=0$, i.e., no external field and vanishing order parameter. Hence the results of this subsection are applicable immediately to the disordered phase. But even the dynamics in the ordered phase will map onto (4.2d) within the framework of our inhomogeneous mean-field theory to be developed in Sec. V A.

Of course, for $\bar{\psi}_{i}^{\alpha}=h=0$, Eqs. (2.5a) and (2.5b) are identical and the nonlinear terms proportional to $b-v$ and $v$ disappear. The resulting equation of motion is most succinctly represented in terms of the Fouriertransformed quantities

$$
u_{i}^{\alpha}(\omega)=\int e^{i \omega t} u_{i}^{\alpha}(t) d t
$$

etc. With the goal of computing the dynamical susceptibility, we introduce a time-dependent external field $h_{i}^{\alpha}(t)$ and finally get

$$
\sum_{j} G_{0}^{-1}{ }_{i j}(\omega) u_{j}^{\alpha}(\omega)-\lambda \sum_{i_{D}} \delta_{i i_{D}} u_{i}^{\alpha}(\omega)=h_{i}^{\alpha}(\omega)+r_{i}^{\alpha}(\omega)
$$

where we have defined the free dynamical propagator 


$$
G_{0}^{-1}(\mathbf{q}, \omega)=a+c q^{2}-I \omega(\omega+i \gamma)
$$

As a consequence of (4.4a) and (4.2b), the thermal average of $u_{i}^{\alpha}$ obeys

$$
\left\langle u_{i}^{\alpha}(\omega)\right\rangle=\sum_{j} G_{0 i j}(\omega) h_{j}^{\alpha}(\omega)+\sum_{j, k} G_{0 i j}(\omega) \Phi_{j k}\left\langle u_{k}^{\alpha}(\omega)\right\rangle .
$$

Here we have introduced a general defect potential $\Phi_{i j}$, which in the special case of pointlike defects introduced in Eq. (2.4a) reads

$$
\Phi_{i j}=\lambda \delta_{i j} \sum_{i_{D}} \delta_{i i_{D}}
$$

According to Eq. (4.5), the dynamical susceptibility, defined by

$$
G_{i j}^{\alpha \beta}(\omega)=\frac{\partial\left\langle u_{i}^{\alpha}(\omega)\right\rangle}{\partial h_{j}^{\beta}(\omega)}=G_{i j}(\omega) \delta^{\alpha \beta},
$$

is diagonal in $\alpha$ and $\beta$ and obeys the equation

$$
G_{i j}(\omega)=G_{0 i j}(\omega)+\sum_{k, l} G_{0 i k}(\omega) \Phi_{k l} G_{l j}(\omega)
$$

Next, we define the Fourier-transformed response function

$$
G_{\mathbf{k k}^{\prime}}(\omega)=\frac{1}{N} \sum_{1 \leq i, j \leq N} G_{i j}(\omega) e^{-i\left(\mathbf{a}_{i} \cdot \mathbf{k}-\mathbf{a}_{j} \cdot \mathbf{k}^{\prime}\right)} .
$$

The corresponding equation for the susceptibility reads

$$
G_{\mathbf{k k}^{\prime}}(\omega)=G_{0}(\mathbf{k}, \omega) \delta_{\mathbf{k k}^{\prime}}+G_{0}(\mathbf{k}, \omega) \sum_{\mathbf{k}^{\prime \prime}} \Phi_{\mathbf{k k}^{\prime \prime}} G_{\mathbf{k}^{\prime \prime} \mathbf{k}^{\prime}}(\omega)
$$

with the Fourier-transformed defect potential

$$
\Phi_{\mathbf{k k}^{\prime}}=\frac{1}{N} \sum_{1 \leq i, j \leq N} \Phi_{i j} e^{-i\left(\mathbf{a}_{i} \cdot \mathbf{k}-\mathbf{a}_{j} \cdot \mathbf{k}^{\prime}\right)}
$$

Before we proceed we would like to note that the present theory can also be formulated within the standard quantum-mechanical lattice dynamics. If the fourth-order nonlinear terms are treated in a Hartree approximation, one ends up with a temperature-dependent harmonic restoring force and the retarded Green's function obeys an equation precisely of the type (4.10).

All physical quantities, as, for instance, $G_{\mathbf{k k}^{\prime}}(\omega)$, have to be averaged over the random spatial defect configurations. Since the positions of the impurities are quenched, these averages have to be performed after the thermodynamic averages. Now we define this configurational average. Conside a single defect $D_{j}$; the configurational average means placing $D_{j}$ at each of the $N$ possible sites $i_{D_{j}}$ and weight each result by $1 / N$. This procedure then has to be repeated for all the $N_{D}$ defects. Hence we arrive at the following definition of the configurational average:

$$
\left\langle\langle\cdots\rangle=\prod_{j=1}^{N_{D}}\left(\frac{1}{N} \sum_{i_{D_{j}}=1}^{N}\right) \cdots\right.
$$

To find the configurational average of $G_{\mathbf{k k}^{\prime}}(\omega)$, one has to iterate Eq. (4.10):

$$
\begin{aligned}
G_{\mathbf{k k}^{\prime}}(\omega)= & G_{0}(\mathbf{k}, \omega) \delta_{\mathbf{k k}^{\prime}}+G_{0}(\mathbf{k}, \omega) \Phi_{\mathbf{k k}^{\prime}} G_{0}\left(\mathbf{k}^{\prime}, \omega\right) \\
& +G_{0}(\mathbf{k}, \omega) \sum_{\mathbf{k}^{\prime \prime}} \Phi_{\mathbf{k k}^{\prime \prime}} G_{0}\left(\mathbf{k}^{\prime \prime}, \omega\right) \Phi_{\mathbf{k}^{\prime \prime} \mathbf{k}^{\prime}} G_{0}\left(\mathbf{k}^{\prime}, \omega\right) \\
& +\cdots
\end{aligned}
$$

The configurational average restores the translational symmetry:

$$
\left\langle\left\langle G_{\mathbf{k k}^{\prime}}(\omega)\right\rangle\right\rangle=G(\mathbf{k}, \omega) \delta_{\mathbf{k k}^{\prime}} .
$$

The evaluation of (4.14) from (4.13) requires the calculation of the configurational average of products of $\Phi_{\mathbf{k k}^{\prime}}$. For instance,

$$
\left\langle\left\langle\Phi_{\mathbf{k k}^{\prime}}\right\rangle\right\rangle=n \lambda \delta_{\mathbf{k k}^{\prime}}
$$

and

$$
\left\langle\left\langle\Phi_{\mathbf{k k}^{\prime \prime}}, \Phi_{\mathbf{k}^{\prime \prime} \mathbf{k}^{\prime}}\right\rangle\right\rangle=\lambda^{2}\left[\frac{n}{N} \delta_{\mathbf{k k}^{\prime}}+n^{2} \delta_{\mathbf{k k}^{\prime \prime}} \delta_{\mathbf{k}^{\prime \prime} \mathbf{k}^{\prime}}\right) .
$$

If double occupancy of sites by the defects is forbidden, Eq. $(4.15 b)$ is to be replaced by ${ }^{40}$

$$
\left\langle\left\langle\Phi_{\mathbf{k k}^{\prime \prime}} \Phi_{\mathbf{k}^{\prime \prime} \mathbf{k}^{\prime}}\right\rangle=\lambda^{2}\left(\frac{n-n^{2}}{N} \delta_{\mathbf{k k}^{\prime}}+n^{2} \delta_{\mathbf{k k}^{\prime \prime}} \delta_{\mathbf{k}^{\prime \prime} \mathbf{k}^{\prime}}\right)\right. \text {. }
$$

There exists an elaborate and widely used diagrammatic representation of the above described perturbation theory for potential scattering in disordered systems (see Refs. 40 and 41). Its basic ingredients are depicted in Fig. 2 containing the propagator, the defect potential, and the impurity concentration. In Fig. 3 we sketch the diagrams for the (full) configurationally averaged propagator $G(\mathbf{q}, \omega)$ up to the third order in $\lambda$ (three dashed lines). We remark that triangles, as in Figs. 3(d) and 3(f)-3(i), imply wave-vector integration over the free propagator within the triangles; the analytic expression corresponding to Fig. 3(d), for example, reads

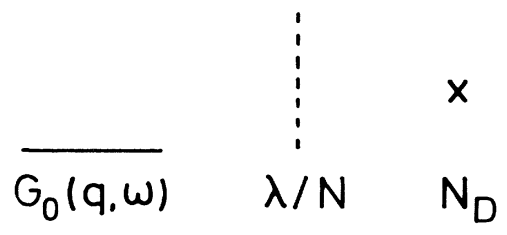

FIG. 2. Fundamental ingredients for the diagrammatic representation of the perturbation theory. 


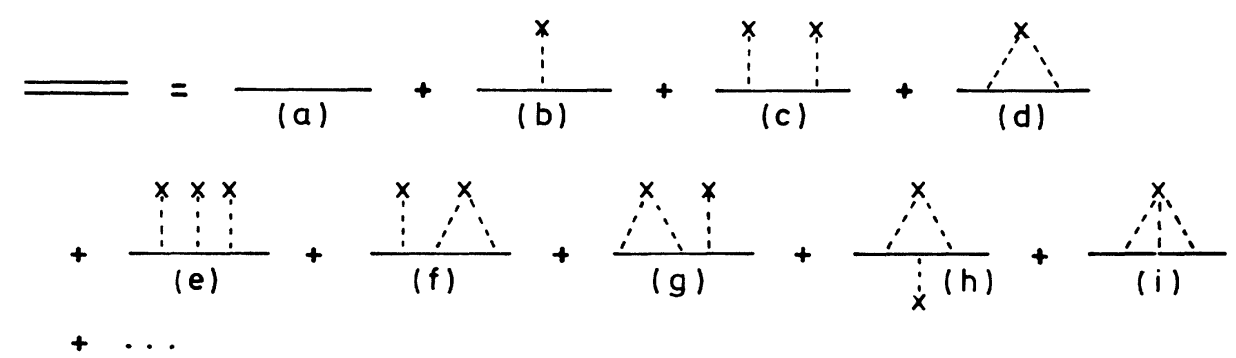

FIG. 3. Diagrams up to the third order in $\lambda$ for the averaged propagator $G(\mathbf{q}, \omega)$.

$G_{0}(\mathbf{q}, \omega) N_{D}(\lambda / N)^{2} \sum_{\mathbf{k}} G_{0}(\mathbf{k}, \omega) G_{0}(\mathbf{q}, \omega)$.

Figure 4 (a) then illustrates how a summation of this perturbation series can be achieved in familiar manner by a Dyson equation yielding

$$
G^{-1}(\mathbf{q}, \omega)=G_{0}^{-1}(\mathbf{q}, \omega)-\Sigma(\mathbf{q}, \omega),
$$

where the self-energy insertion $\Sigma(\mathbf{q}, \omega)$ consists of the one-particle irreducible diagrams [Fig. 4(b)]. These selfenergy contributions can now be grouped according to the number of crosses, which transforms the perturbation theory into one where the expansion parameter $\lambda$ is effectively replaced by the impurity concentration $n$.

We shall restrict our approximation to taking into account only those terms that linearly depend on $n$ (Fig. 5), known as the single-site approximation. The resultant series can once again be evaluated easily via the geometric sum formula

$$
\begin{aligned}
& \Sigma^{\prime}(\omega)=n \lambda+n \lambda^{2} \frac{1}{N} \sum_{\mathbf{k}} G_{0}(\mathbf{k}, \omega) \\
& +n \lambda^{3}\left[\frac{1}{N} \sum_{\mathbf{k}} G_{0}(\mathbf{k}, \omega)\right]^{2}+\cdots \\
& =\frac{n \lambda}{1-(\lambda / N) \sum_{\mathbf{k}} G_{0}(\mathbf{k}, \omega)} . \\
& ==\frac{(\mathrm{a})}{=}
\end{aligned}
$$

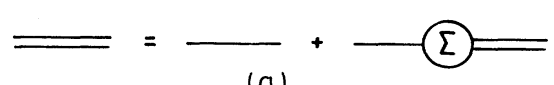

(2)

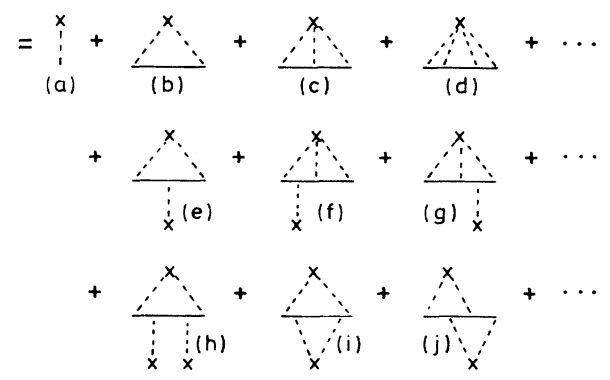

(b)

FIG. 4. (a) Dyson equation. (b) Self-energy insertions to fourth order in $\lambda$.
We remark that we have now arrived at a stage where the phonon response function (4.16) together with (4.17) has the form of a double fraction expansion. A different derivation of Eqs. (4.16) and (4.17) is given in Appendix $B$, which is based on the exact one-defect correlation function and an average thereof.

\section{B. Correlation function above $T_{c}(n)$ and emergence of a central peak}

Equations (4.16) and (4.17) of the preceding subsection can be applied immediately to the phonon response function at sufficiently high temperatures, such that $\bar{\psi}_{i}^{\alpha}$ vanishes, using Eq. (4.4b) for the bare dynamical susceptibility $G_{0}(\mathbf{q}, \omega)$. First, we state the important fact that $G^{-1}(0,0)=0$ in general marks an instability; i.e., there is a proper phase transition at a certain concentrationdependent temperature $T_{c}(n)$, defined through

$$
a_{c}(n)\left[1-\frac{\lambda}{N} \sum_{\mathrm{k}} \frac{1}{a_{c}(n)+c k^{2}}\right]=n \lambda,
$$

or equivalently

$$
\lambda=\frac{a_{c}(n)}{n+(1 / N) \sum_{\mathbf{k}}\left\{1+\left[c / a_{c}(n)\right] k^{2}\right\}^{-1}} .
$$

Now the focus of our discussion will be the vicinity of this critical point, which is induced by the softening action of a finite concentration of defects. In order to determine the critical value of the Ginzburg-Landau parameter $a_{c}(n)=a^{\prime}\left[T_{c}(n)-T_{c}^{0}\right]$ and therefrom $T_{c}(n)$, we simply have to evaluate the following integral in arbitrary dimension $d$ :

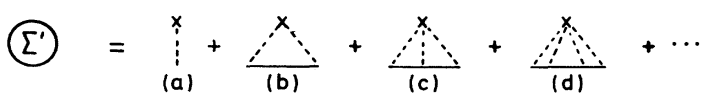

FIG. 5. Self-energy contributions in the single-site approximation. 
$\frac{1}{N} \sum_{\mathbf{k}} \frac{1}{a+c k^{2}}=\frac{V}{N a}\left(\frac{a}{c}\right)^{d / 2} \int \frac{d^{d} p}{(2 \pi)^{d}} \frac{1}{1+p^{2}}$.

Introducing a cutoff $\Lambda$ corresponding to the boundary of the Brillouin zone, we get

$$
2 \int_{0}^{\Lambda \xi^{>}} \frac{d p}{2 \pi} \frac{1}{1+p^{2}}=\frac{1}{\pi} \arctan \Lambda \xi^{>} \rightarrow \frac{1}{2} \text { as } \Lambda \rightarrow \infty
$$

for $d=1$;

$$
2 \pi \int_{0}^{\Lambda \xi^{>}} \frac{d p}{(2 \pi)^{2}} \frac{p}{1+p^{2}}=\frac{1}{4 \pi} \ln \left[1+\left(\Lambda \xi^{>}\right)^{2}\right],
$$

for $d=2$; and

$$
4 \pi \int_{0}^{\Lambda \xi^{>}} \frac{d p}{(2 \pi)^{3}} \frac{p^{2}}{1+p^{2}}=\frac{1}{2 \pi^{2}}\left[\Lambda \xi^{>}-\arctan \left(\Lambda \xi^{>}\right)\right],
$$

for $d=3$.

From (4.18a) we recover in the limit $n \rightarrow 0$ the condition for the single-defect local transition temperature $T_{c}^{l}$ considered in Sec. III:

$$
1-\frac{\lambda}{N} \sum_{\mathbf{k}} \frac{1}{a_{c}(0)+c k^{2}}=0,
$$

with $a_{c}(0)=a^{\prime}\left(T_{c}^{l}-T_{c}^{0}\right)$. For finite concentration an expansion of Eq. (4.18a) together with (4.20) leads to

$$
\begin{aligned}
T_{c}(n)= & T_{c}^{l}+\frac{n}{a^{\prime}} /\left[-a_{c}(0) \frac{\partial}{\partial a_{c}(0)} \frac{1}{N} \sum_{\mathbf{k}} \frac{1}{a_{c}(0)+c k^{2}}\right) \\
& +O\left(n^{2}\right) .
\end{aligned}
$$

For $d \geq 2$, a certain minimal defect strength is required to allow for a solution of Eq. (4.18) and the ensuing defect-induced phase transition. In the one-dimensional model, however, Eq. (4.18) for the critical temperature can be solved for any value of the defect strength, and we may even take the limit $\Lambda \rightarrow \infty$, with the result

$$
\begin{aligned}
t_{c}(n) & =\frac{T_{c}(n)-T_{c}^{0}}{T_{c}^{0}} \\
& =\left(\frac{U_{0}}{4\left(a^{\prime} T_{c}^{0} c\right)^{1 / 2}}\right)^{2}\left[1+\left(1+n^{\prime} \frac{16 c}{U_{0}}\right)^{1 / 2}\right]^{2},
\end{aligned}
$$

where we have introduced $U_{0}=a_{0}^{d} \lambda$ and the impurity concentration per unit volume, $n^{\prime}=N_{D} / V$. To first order in $n^{\prime}$, we find

$$
T_{c}(n)=T_{c}^{l}+\frac{2 n^{\prime} U_{0}}{a^{\prime}}+O\left(n^{\prime 2}\right)
$$

showing the close relationship between the local condensation temperature (3.6) of the single-defect system and the defect-induced transition temperature of the crystal with infinitely many randomly distributed impurities. For higher dimensions, Eq. (4.18) is more difficult to solve, as the cutoff $\Lambda$ has to remain finite. The resulting expressions contain rather complicated functions of the temperature, and an inversion similar to Eq. (4.22a) is impossible. In three dimensions, Eq. (4.18a) can be solved elementarily under the condition $\Lambda \sqrt{c / a} \gg 1$. Then one finds for the minimal defect strength $U_{0}^{\min }=2 \pi^{2} c / \Lambda$, and, if this is exceeded,

$$
T_{c}^{l}=T_{c}^{0}+\frac{16 \pi^{2} c^{3}}{a^{\prime}\left(U_{0}^{\min }\right)^{2}}\left(1-\frac{U_{0}^{\min }}{U_{0}}\right)^{2}
$$

and

$$
T_{c}(n)=T_{c}^{l}+\frac{4 \pi n^{\prime}}{\left(a^{\prime} / c\right)^{3 / 2}\left(T_{c}^{l}-T_{c}^{0}\right)^{1 / 2}} .
$$

We remark that in deriving the dependence on the lattice constant $a_{0}$ (hidden in the parameters $U_{0}$ [Eq. (3.5)] and $n^{\prime}$ in (4.22) and (4.23)) one has to be aware that $\Lambda^{d} \approx\left(\pi / a_{0}\right)^{d}=\pi^{d} N / V$. From Eqs. (4.21)-(4.23b) it is apparent that $T_{c}(n)$ is higher than $T_{c}^{l}$, but very close to it for small concentrations. The value of $T_{c}^{l}$ depends only on the strength of a single defect and may be much higher than $T_{c}^{0}$.

Using Eqs. (4.19), we can explicitly perform the wavenumber integration over the free propagator as required in (4.17),

$$
I_{d}(\omega)=\frac{\lambda}{N} \sum_{\mathbf{k}} G_{0}(\mathbf{k}, \omega)
$$

reading, in one, two, and three dimensions,

$$
\begin{aligned}
I_{1}(\omega) & =\frac{U_{0}}{2 \pi i \sqrt{a c}} \frac{1}{\sqrt{1-(I / a) \omega(\omega+i \gamma)}} \ln \frac{\sqrt{1-(I / a) \omega(\omega+i \gamma)}+i \Lambda \xi^{>}}{\sqrt{1-(I / a) \omega(\omega+i \gamma)}-i \Lambda \xi^{>}}, \\
& \rightarrow \frac{U_{0}}{2 \sqrt{a c}} \frac{1}{\sqrt{1-(I / a) \omega(\omega+i \gamma)}} \text { as } \Lambda \rightarrow \infty, \\
I_{2}(\omega) & =\frac{U_{0}}{4 \pi c} \ln \left[1+\frac{\left(\Lambda \xi^{>}\right)^{2}}{1-(I / a) \omega(\omega+i \gamma)}\right], \\
I_{3}(\omega) & =\frac{U_{0}}{2 \pi^{2} c \xi^{>}}\left[\Lambda \xi^{>}-\frac{1}{2 i} \sqrt{1-(I / a) \omega(\omega+i \gamma)} \ln \frac{\sqrt{1-(I / a) \omega(\omega+i \gamma)}+i \Lambda \xi^{>}}{\sqrt{1-(I / a) \omega(\omega+i \gamma)}-i \Lambda \xi^{>}}\right] .
\end{aligned}
$$


Inserting (4.24) into Eqs. (4.16) and (4.17) yields our analytical results (note that $n \lambda=n^{\prime} U_{0}$ ) for the dynamical phonon response function. Figure 6 shows the phononphonon correlation function $D(\mathbf{q}, \omega)$ [Eq. (2.14)] for several temperatures closely above $T_{c}(n)$ in three dimensions for comparatively high phonon damping coefficient $\gamma$ and low impurity concentration $n$, using GinzburgLandau coefficients that seem reasonable for $\mathrm{SrTiO}_{3}$ (Table I). Here $t=\left(T-T_{c}^{0}\right) / T_{c}^{0}$ and $t=0.6$ corresponds to $T=T_{c}(n)$. We find the following qualitative features: A rather broad phonon peak, which softens when the tmeperature is lowered, and a narrow central peak in the immediate vicinity of $T_{c}(n)$, obviously a dynamical precursor of the defect-induced phase transition.

We remark that also different scenarios can be achieved when the concentration is higher and the damping lower. In this case a pronounced phonon impurity band is revealed stemming from the localized optic phonons found in the one-defect system. Their softening at $T=T_{c}^{l}$ corresponds to the local condensation taking place in the vicinity of the impurities; hence we expect that the origin of the dynamical central peak can be found in the softening of the impurity band.

For a more detailed physical understanding of the mechanism that produces the dynamical central peak, we now keep the temperature fixed $(t=0.61)$ and vary the phonon damping constant $\gamma$ (Fig. 7). We see that for low damping the impurity band is located at a finite frequency [approximately given by Eq. (3.12b) in the onedimensional case], which becomes more and more overdamped when $\gamma$ is raised. Eventually, this mode will display a relaxator behavior critically slowing down for $T \rightarrow T_{c}(n)$ and thereby producing a central peak. Figure 6 then describes a scenario where the damping $\gamma$ is

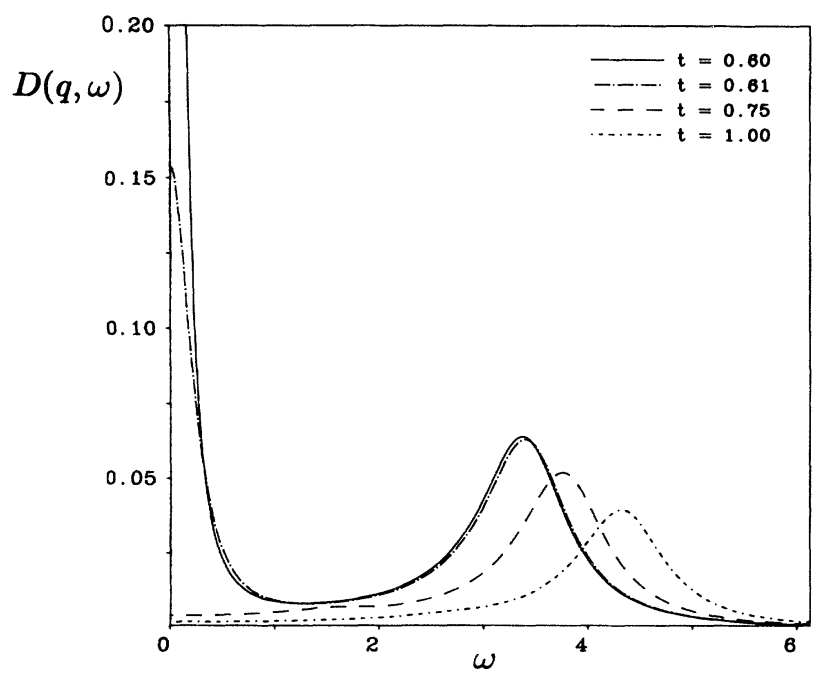

FIG. 6. Phonon-phonon correlation function $D(\mathbf{q}, \omega)$ above $T_{c}(n)$ for several temperatures. $T=T_{c}(n)$ corresponds to $t=0.6$ and $T=T_{c}^{0}$ to $t=0\left(\omega\right.$ in units $\left.10^{-3} \mathrm{eV} / \hbar\right)$.

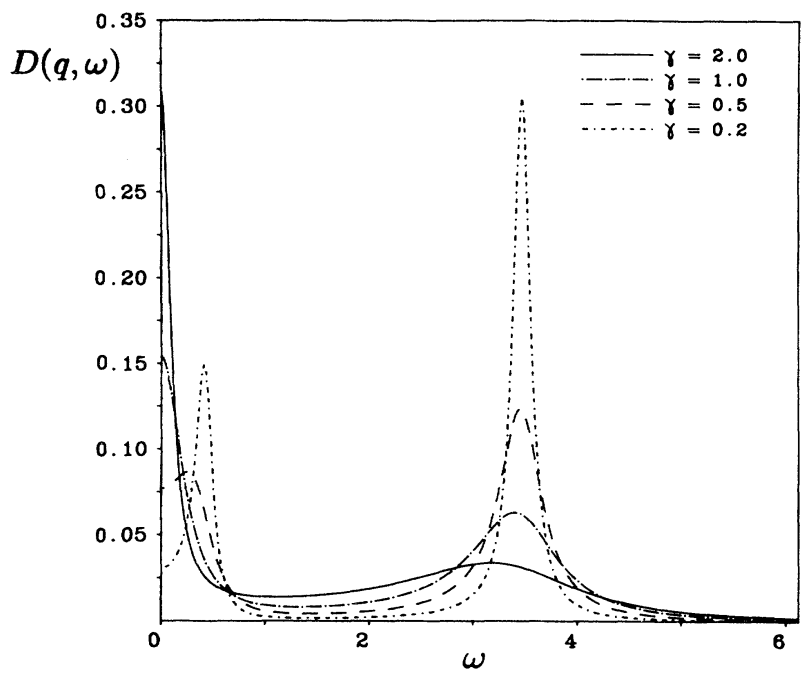

FIG. 7. Phonon-phonon correlation function $D(\mathbf{q}, \omega)$ at fixed temperature $(t=0.61)$ for varying phonon damping coefficient $\gamma\left(\omega\right.$ and $\gamma$ in units $\left.10^{-3} \mathrm{eV} / \hbar\right)$.

higher and the defect concentration $n$ low enough in order that the impurity band has a negligible weight in comparison to the soft bulk-phonon peak at higher temperatures. Only when the critical point at $T_{c}(n)$ is approached and the impurity mode has already changed its character from an oscillator to a pure relaxator does it appear as a narrow central peak in the dynamical correlation function.

For the one-dimensional case, we can easily confirm these results analytically. In the limit $\omega \rightarrow 0$, we find that the response function is roughly proportional to $(\omega-i \Gamma)^{-1}$ with

$\Gamma=\frac{2 a}{I \gamma} \frac{a-\left(U_{0} / 2\right) \sqrt{a / c}-n^{\prime} U_{0}}{3 a-U_{0} \sqrt{a / c}-n^{\prime} U_{0}} \propto T-T_{c}(n)$.

Now we can formulate a quantitative criterion for the impurity band becoming overdamped and thus producing a dynamical central peak: This will certainly be the case if the width $\Gamma$ of the relaxator peak is about half (the factor 2 being somewhat arbitrary) the frequency of the impurity band $\omega_{l}$. Figure 7 confirms this assertion.

We remark that these results are in qualitative accord with those of Refs. 31-34 and with molecular-dynamics simulations by Weyrich, Wiesen, and Siems ${ }^{35,36}$ for a oneand two-defect distortive system with periodic boundary conditions in one and two dimensions, inasmuch as those one-defect systems can be compared with the more general random impurity system under discussion here.

\section{STATICS AND DYNAMICS IN THE ORDERED PHASE $T<T_{c}(n)$}

The computation of the order-parameter configuration and the response and correlation functions in the ordered 
phase is, in general, a sophisticated issue, because now the nonlinearities are decisive. One has to expect that they will not just alter the propagators introducing an additional mass term, but also should affect the defect potential strength via a screening effect. These complications must obviously be taken into account by using a self-consistent approach, the simplest possible one presented in Sec. V A. With this background the phonon response and correlation functions can be calculated in a fashion very similar to the one described in Sec. IV, with modified parameters $a$ and $\lambda$. For an $M$-component order parameter, we have to distinguish between the longitudinal and transverse directions with respect to the spontaneously ordered phase. We shall see that the dynamical central peak disappears in the longitudinal part, while it persists in the transverse direction if there is a continuous rotational symmetry. The effect of cubic terms breaking this rotational invariance is investigated also in Sec. V B. Anyway, the finite order parameter will induce a static $\delta(\omega)$-shaped central peak in the dynamical structure function relevant, e.g., for neutron-scattering experiments. However, because of the inhomogeneous static configuration, this peak will have, besides a Braggtype contribution, a finite width in momentum space. These topics will be discussed in the final part of this section.

\section{A. Computation of the order parameter}

In order to actually calculate the order-parameter configuration, we have to return to Eq. (2.5a) for the longitudinal field, applying a constant external field $h_{i}=h$, which eventually is set to zero. Of course, $\bar{\psi}_{i}^{\alpha}=0$ for $\alpha \neq \mathrm{L}$, and Eq. (4.1) using (2.5a) thus reduces to

$$
\sum_{j}\left[G_{0}^{-1}{ }_{i j}+b\left(\bar{\psi}_{i}^{\mathrm{L}}\right)^{2} \delta_{i j}\right] \bar{\psi}_{j}^{\mathrm{L}}-\lambda \sum_{i_{D}} \delta_{i i_{D}} \bar{\psi}_{i}^{\mathrm{L}}=h .
$$

This equation is difficult to solve because of the combined problems of nonlinearity and randomness. Therefore, we develop a self-consistent approximation, an inhomogeneous mean-field theory, based on the following physical ideas: Immediately below $T_{c}(n)$ we expect a situation where the order parameter almost vanishes, reaching markably finite values only in the very surroundings of the softening defects. On the other hand, these hardly overlapping "condensates" will certainly screen and thereby weaken the defect potential strength. Far below $T_{c}(n)$, especially when the bulk transition temperature $T_{c}^{0}$ of the pure crystal is approached, these local effects will become far less important, the configuration being dominated by a global, more or less homogeneous orderparameter state. These considerations show that we have to distinguish between the "pure" lattice points and the impurity sites with their nearby neighbors. Obviously, the simplest ansatz, illustrated in Fig. 8 (compare Fig. 1), for the order parameter, taking into account the basic physics, is

$$
\bar{\psi}_{i}^{\mathrm{L}}=A+B \sum_{i_{D}} \delta_{i i_{D}} .
$$

Here $A$ is the polarization of the pure sites and $A+B$ of

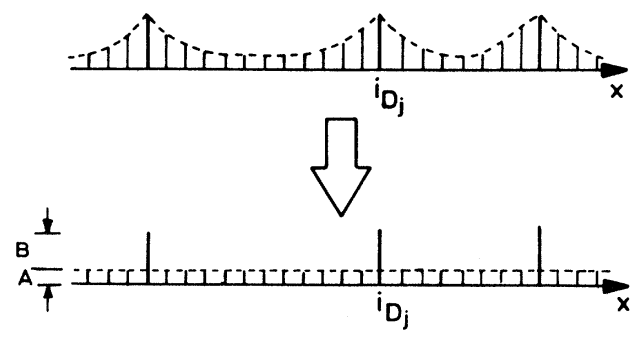

FIG. 8. Schematic illustration of the order-parameter configuration according to the approximation (5.2).

the defect sites. Taking the average over the defect configurations [Eq. (4.12)] then yields for the average order parameter, which of course is independent of $i$,

$$
\left\langle\left\langle\bar{\psi}^{\mathrm{L}}\right\rangle\right\rangle=A+n B \text {. }
$$

For the square of the local order parameter, as needed in (5.1), we obtain, from Eq. (5.2),

$$
\left(\bar{\psi}_{i}^{\mathrm{L}}\right)^{2}=A^{2}+\left[(A+B)^{2}-A^{2}\right] \sum_{i_{D}} \delta_{i i_{D}} .
$$

Inserting (5.4) into (5.1), the space-dependent order parameter $\bar{\psi}_{i}^{L}$ obeys

$$
\sum_{j} \widetilde{G}_{0}^{-1}{ }_{i j} \bar{\psi}_{j}^{\mathrm{L}}-\tilde{\lambda} \sum_{i_{D}} \delta_{i i_{D}} \bar{\psi}_{i}^{\mathrm{L}}=h
$$

Here the nonlinearity is absorbed entirely into modified coefficients:

$$
\begin{aligned}
& \widetilde{G}_{0}^{-1}(\mathbf{q})=\widetilde{a}+c q^{2}, \\
& \widetilde{a}=a+b A^{2}, \\
& \widetilde{\lambda}=\lambda-b B(2 A+B) .
\end{aligned}
$$

Hence, indeed, the inhomogeneous order parameter leads to a modified Ginzburg-Landau coefficient $a$ [Eq. (5.6b)] and screens the defect potential [Eq. (5.6c)].

The linear equation (5.5) for the order parameter $\bar{\psi}_{i}^{L}$ can be used to determine the defect-averaged order parameter $\left\langle\left\langle\bar{\psi}^{\mathrm{L}}\right\rangle\right\rangle$ in analogy to Sec. IV A. If we introduce the Fourier transform of $\bar{\psi}_{i}^{\mathrm{L}}$,

$$
\bar{\psi}_{\mathbf{q}}^{\mathrm{L}}=\frac{1}{N} \sum_{i=1}^{N} \bar{\psi}_{i}^{\mathrm{L}} e^{-i \mathbf{q} \cdot \mathbf{a}_{i}}
$$

we get, from (5.5),

$$
\bar{\psi}_{\mathbf{q}}^{\mathrm{L}}=h \widetilde{G}_{0}(\mathbf{0}) \delta_{\mathbf{q}, 0}+\widetilde{G}_{0}(\mathbf{q}) \sum_{\mathbf{k}} \widetilde{\Phi}_{\mathbf{q k}} \bar{\psi}_{\mathbf{k}}^{\mathrm{L}},
$$

which can be iterated. Already, the first step

$$
\begin{aligned}
\bar{\psi}_{\mathbf{q}}^{\mathrm{L}}= & h \widetilde{G}_{0}(\mathbf{0}) \delta_{\mathrm{q}, 0}+\widetilde{G}_{0}(\mathbf{q}) \widetilde{\Phi}_{\mathrm{q}, 0} h \widetilde{G}_{0}(\mathbf{0}) \\
& +\widetilde{G}_{0}(\mathbf{q}) \sum_{\mathbf{k}} \widetilde{\Phi}_{\mathrm{qk}} \widetilde{G}_{0}(\mathbf{k}) \widetilde{\Phi}_{\mathbf{k}, 0} h \widetilde{G}_{0}(\mathbf{0})+\cdots,
\end{aligned}
$$


and the similarity of Eqs. (5.8) and (4.10) reveals that a similar perturbation expansion for $\left\langle\left\langle\bar{\psi}_{\mathbf{q}}^{\mathrm{L}}\right\rangle\right\rangle=\left\langle\left\langle\bar{\psi}^{\mathrm{L}}\right\rangle\right\rangle \delta_{\mathrm{q}, 0}$ can be introduced as in Sec. IV [see Fig. 9(a) for the elements of the graphical representation and Fig. 9(b) for a number of diagrams and their effective summation via a self-energy insertion $\Sigma$ ]. In single-site approximation we obtain the following final result:

$$
\frac{\left\langle\left\langle\bar{\psi}^{\mathrm{L}}\right\rangle\right\rangle}{h}=\left(\widetilde{a}-\frac{n \tilde{\lambda}}{1-(\tilde{\lambda} / N) \sum_{\mathbf{k}}\left(\widetilde{a}+c k^{2}\right)^{-1}}\right]^{-1},
$$

for the averaged order parameter in the presence of an external field. In order that the value of the static displacement field remains finite even in the limit $h \rightarrow 0$, i.e., a spontaneous order parameter may exist, we find the condition

$$
\widetilde{\lambda}=\frac{\widetilde{a}}{n+(1 / N) \sum_{\mathbf{k}}\left[1+(c / \widetilde{a}) k^{2}\right]^{-1}} .
$$

For the determination of the averaged order parameter, we need a second relationship, which is obtained by taking the configurational average of Eq. (5:5):

$$
\widetilde{G}_{0}^{-1}(0)\left\langle\left\langle\bar{\psi}^{\mathrm{L}}\right\rangle\right\rangle-n(A+B) \tilde{\lambda}=h \text {. }
$$

Equations (5.10) and (5.12), together with (5.3), constitute the basic equations for the order parameter of this random system. From these one can determine $\left\langle\left\langle\bar{\psi}^{L}\right\rangle\right\rangle$ as a function of $h$ and $T$. In the limit $h=0$, instead of (5.10), Eq. (5.11) may be used.

Now we discuss some of the consequences of Eqs. (5.11) and (5.12), which allow the determination of the

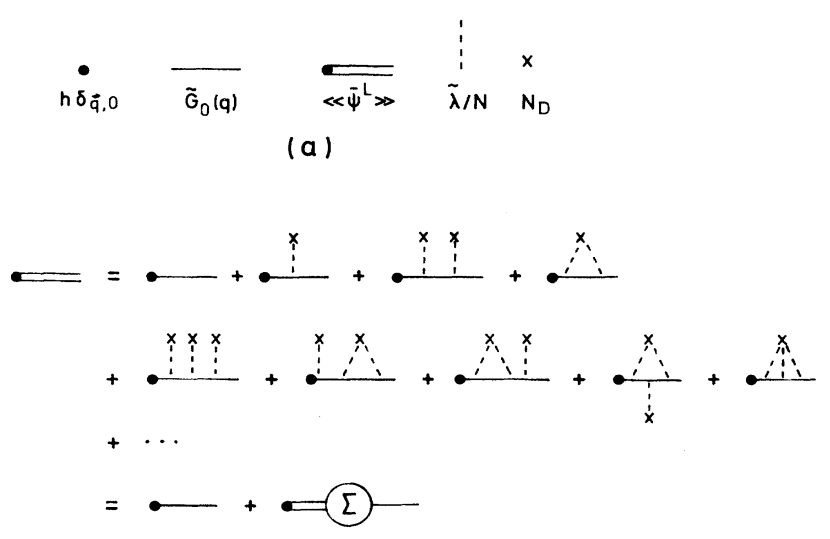

(b)

FIG. 9. (a) Elements of the diagrammatic representation of the order-parameter perturbation theory. (b) Diagrams up to the third order in $\lambda$ for the averaged order-parameter $\left\langle\left\langle\bar{\psi}^{L}\right\rangle\right\rangle$ and their summation via a self-energy insertion $\Sigma$. In single-site approximation only the diagrams of Fig. 5 are summed up.

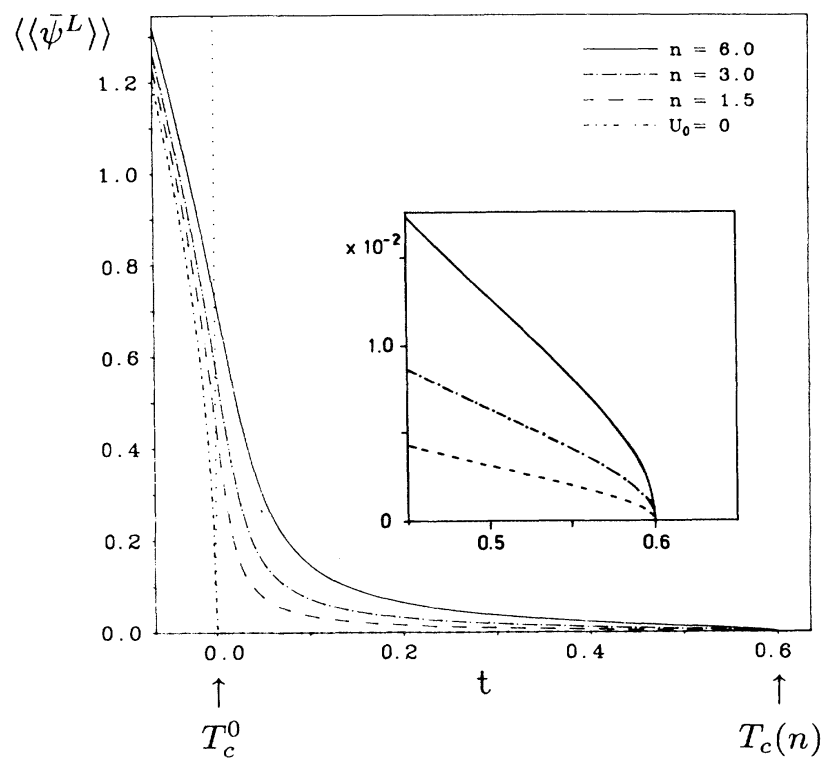

FIG. 10. Mean order parameter $\left\langle\left\langle\bar{\psi}^{\mathrm{L}}\right\rangle\right\rangle=A+n B$ (in units $10^{-2}$ ) for different defect concentrations $n$ ( $n$ in units $10^{-5}$ ) [and also slightly different defect strengths $U_{0}$, such that $t_{c}(n)=0.6$ remains fixed]. The pure system's $\left(U_{0}=0\right)$ order parameter is also displayed.

averaged order parameter. First of all, it is reassuring that Eq. (5.11) for $A=B=0$ and $a=a_{c}(n)$ coincides with Eq. (4.18b), and consequently the order parameter sets in at the transition temperature determined from the instability of the symmetric phase.

Inserting (5.11) into (5.12) leads to a nonlinear equation for the parameter $A$, which in limiting cases can be solved analytically and otherwise numerically. Figure 10 depicts the three-dimensional mean order parameter (5.3) for different defect concentrations $n$. Very close to $T_{c}(n)$ the coexistence curve starts off with the usual mean-field exponent $\beta=\frac{1}{2}$ (see the inset in Fig. 10), the order parameter being roughly proportional to $n$. When approaching the bulk transition temperature of the pure system $T_{c}^{0}$, however, the character of the static configuration qualitatively changes into more or less the state of the pure system (also shown in Fig. 10 for comparison), with slightly an enhanced value. Here the homogeneous part $A$ of Eq. (5.2) clearly dominates, while in the vicinity of the critical point the "local" contribution $B$ is by far important.

The dependence of $t_{c}(n)=\left[T_{c}(n)-T_{c}^{0}\right] / T_{c}^{0}$ on defect strength $\lambda$ (or $U_{0}$ ) and concentration $n$ can be read off from Fig. 11. Thus, for $\lambda / b=0.069$ and $n=6.0$ $\times 10^{-5}, 1.5 \times 10^{-5}$, and $0.1 \times 10^{-5}$, the value of $t_{c}(n)$ is very close to $t_{c}(0)=\left(T_{c}^{\prime}-T_{c}^{0}\right) / T_{c}^{0}$, and then the defectinduced transition temperature is approximately given by the local condensation temperature $T_{c}^{l}$ [see Eq. (4.21)]. Yet the shape of the average order parameter changes drastically if the concentration is lowered (Fig. 10). For high concentration (higher than shown in Fig. 10), it has more or less the familiar square-root shape. However, for 


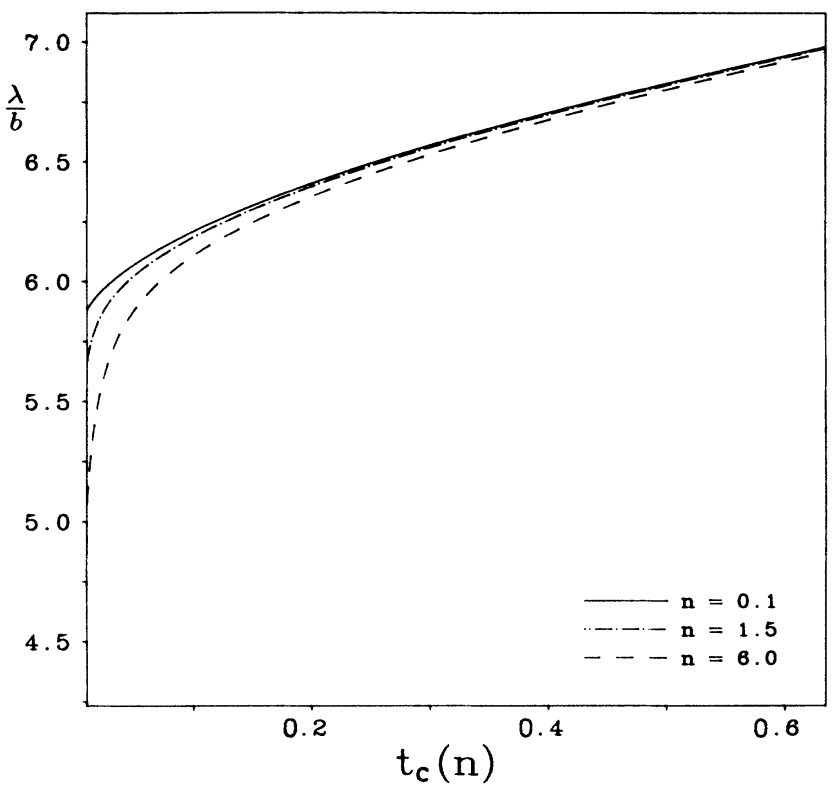

FIG. 11. Defect strength $\lambda / b$ (in units $10^{-2}$ ) vs the defectinduced transition temperature $t_{c}(n)=\left[T_{c}(n)-T_{c}^{0}\right] / T_{c}^{0}$ (or vice versa) for the concentrations $n=0.1 \times 10^{-5}, 1.5 \times 10^{-5}$, and $6.0 \times 10^{-5}$

small concentration the order parameter sets in with a small amplitude at $T_{c}(n)$, stays minute in the whole region $T_{c}^{0}<T<T_{c}(n)$, and gets amplified near $T_{c}^{0}$. For the smallest concentration shown in Fig. 10, and even more pronounced for smaller ones, the order parameter is almost zero for $T_{c}^{0}<T<T_{c}(n)$, such that it has the appearance of an order parameter of an ideal pure system $\propto\left(T_{c}^{0}-T\right)^{1 / 2}$, which is slightly rounded at $T_{c}^{0}$.

\section{B. Phonon response function}

Below $T_{c}(n)$ we have to distinguish between the fluctuations parallel to the order parameter, $\alpha=\mathbf{L}$, and in the $M-1$ transverse directions, $\alpha=\mathrm{T}$. Thus

$$
G^{\alpha \beta}(\mathbf{q}, \omega)=G^{\alpha \alpha}(\mathbf{q}, \omega) \delta^{\alpha \beta} .
$$

In order to calculate the response and correlation function in the presence of the spatially inhomogeneous order parameter, we again start from the linearized equation of motion (4.2d) and insert (5.2) into the right-hand side. Including the time-dependent fields $h_{i}^{\alpha}(t)$ then yields relations quite similar to Eq. (4.4a), with modified masses and defect strengths for the longitudinal and transverse directions. The results for the response function therefore take the form (4.16), where in single-site approximation the self-energy is given by (4.17).

For the longitudinal part the renormalized coefficients to be inserted into Eqs. (4.16) and (4.17) are

$$
a_{\mathrm{L}}=a+3 b A^{2} \text {, }
$$

$$
\lambda_{\mathrm{L}}=\lambda-3 b B(2 A+B) .
$$

Therefore, we find, for the longitudinal dynamical susceptibility,

$G_{\mathrm{L}}^{-1}(\mathrm{q}, \omega)=a_{\mathrm{L}}+c q^{2}-I \omega(\omega+i \gamma)-\frac{n \lambda_{\mathrm{L}}}{1-I_{d}^{\mathrm{L}}(\omega)}$,

where in Eqs. (4.24) for the integral $I_{d}^{\mathrm{L}}(\omega)$ the parameters $a$ and $\lambda$ have to be replaced by $a_{\mathrm{L}}$ and $\lambda_{\mathrm{L}}$, respectively. The longitudinal phonon-phonon correlation function $D_{\mathrm{L}}(\mathbf{q}, \omega)$ is displayed in Fig. 12, demonstrating that the dynamical central peak is confined to the critical region very close to $T_{c}(n)$ and disappears again further away from $T_{c}(n)$ because of the temperature dependence in (5.14). This just reflects the fact that in the single-defect system (Sec. III B) the localized mode moves back into the continuum of extended states. ${ }^{32}$

Within the mean-field approximation used here, the longitudinal static susceptibility below $T_{c}(n)$ remains finite for $q \rightarrow 0$. The longitudinal static response function $G_{\mathrm{L}}(\mathrm{q}, 0)$ is plotted for several values of the wave vector $q$ in Fig. 13(a), and also $G_{\mathrm{L}}^{-1}(0,0)$ for different defect concentrations in Fig. 13(b), showing that $G_{\mathrm{L}}(0,0)$ diverges at $T=T_{c}(n)$ and obtains considerable values only in its immediate surroundings. At the bulk transition temperature $T_{c}^{0}$ of the pure crystal, $G_{\mathrm{L}}(\mathbf{0}, 0)$ remains finite, although it may become fairly large for low concentrations. The divergence of the static susceptibility of the pure crystal at $T_{c}^{0}$ is modified into a maximum slightly above $T_{c}^{0}$. As apparent from Fig. 13(a), the divergence of $G_{\mathrm{L}}(0,0)$ is confined to an extremely narrow interval around $T_{c}(n)$ and will be much harder to detect than the remnant of the pure transition near $T_{c}^{0}$.

Now we turn to the transverse response function. The transverse parameters are given by

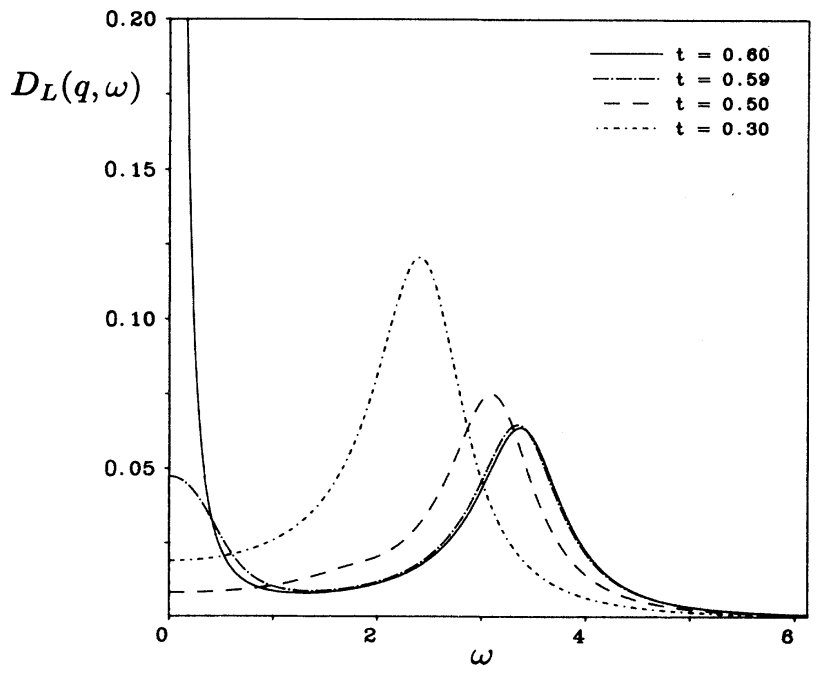

FIG. 12. Longitudinal phonon-phonon correlation function $D_{\mathrm{L}}(\mathbf{q}, \omega)$ below $T_{c}(n)$, for several temperatures (see Fig. 6). 


$$
\begin{aligned}
& a_{\mathrm{T}}=a+(b-v) A^{2}, \\
& \lambda_{\mathrm{T}}=\lambda-(b-v) B(2 A+B) .
\end{aligned}
$$

Thus, in the case of an isotropic system, with $v=0$, they are identical to $\widetilde{a}$ and $\tilde{\lambda}$ of Sec. V A. Because of Eq. (5.11), this implies that the static transverse response function diverges as

$$
G_{\mathrm{T}}(\mathbf{q}, 0) \propto \frac{1}{q^{2}},
$$

in the limit of small $q$, which is nothing but a confirmation of the Goldstone theorem (see, e.g., Ref. 42) for translationally invariant systems with spontaneously
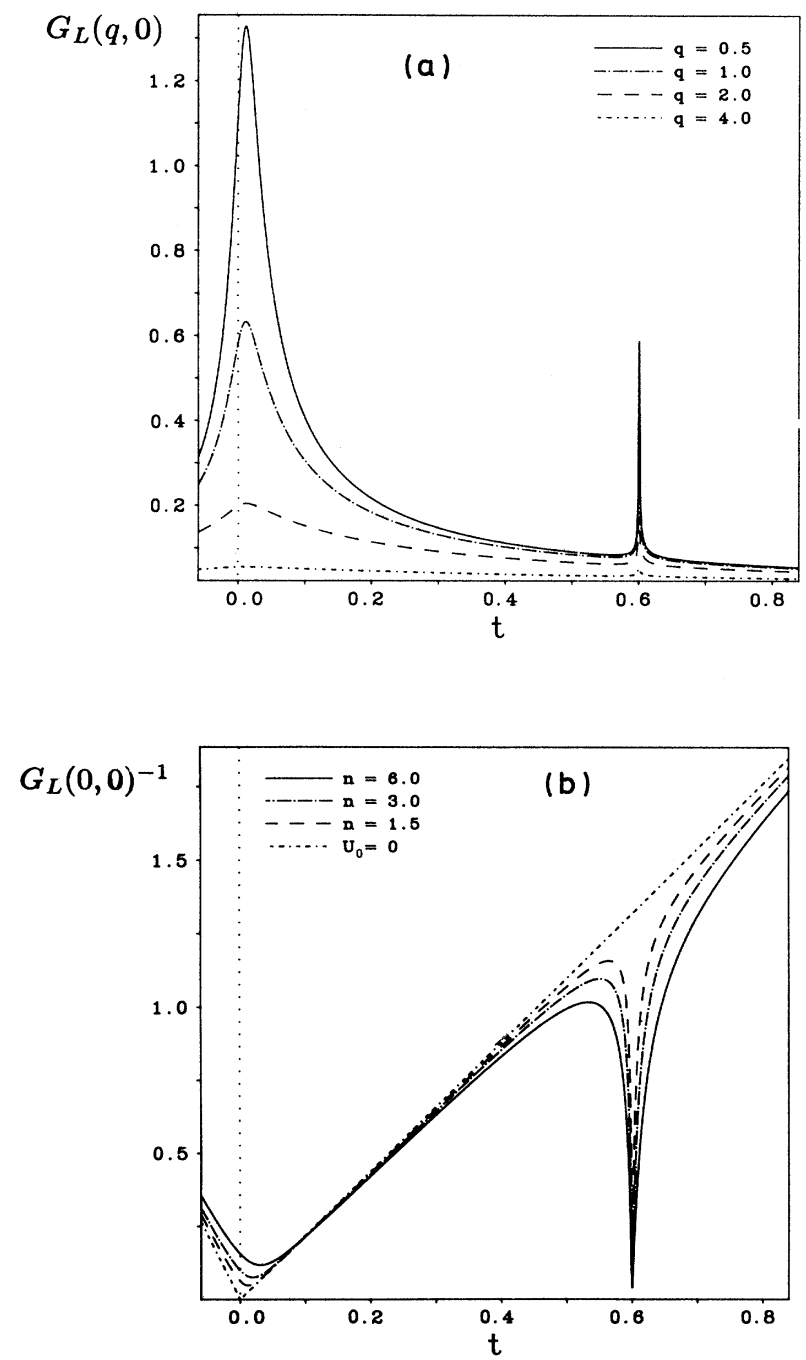

FIG. 13. (a) Longitudinal static susceptibility $G_{\mathrm{L}}(\mathbf{q}, 0)$ for several values of the wave vector $q$ ( $q$ in units $2.8 \times 10^{6} \mathrm{~cm}^{-1}$ ). (b) Inverse longitudinal static susceptibility $G_{\mathrm{L}}^{-1}(0,0)$ for different defect concentrations $n$ ( $n$ in units $10^{-5}$ ). For comparison, the graph for the pure system $\left(U_{0}=0\right)$ is also displayed. broken continuous rotational invariance. Using Eqs. (5.10), (5.6), and (5.15), we see that, for finite uniform external field $h$,

$$
G_{\mathrm{T}}(0,0)=\frac{\bar{\psi}}{h}
$$

a result also known from field theory as a certain WardTakahashi identity. ${ }^{42}$ At the first glance it may seem peculiar that we gain relations of this kind in our highly disordered system. One has to realize, however, that the continuous internal symmetry is not violated by the random defects, while translational invariance is restored by the defect-averaging procedure. Thus it seem reasonable to expect that in a correct treatment of the fluctuations via a renormalization-group analysis, even the longitudinal response function might diverge according to

$$
G_{\mathrm{L}}(\mathbf{q}, 0) \propto \frac{1}{q^{\epsilon}},
$$

where $0<\epsilon=4-d<2 .{ }^{43}$

The general wave-number- and frequency-dependent transverse susceptibility is of the form

$G_{\mathrm{T}}^{-1}(\mathbf{q}, \omega)=a_{\mathrm{T}}+c q^{2}-I \omega(\omega+i \gamma)-\frac{n \lambda_{\mathrm{T}}}{1-I_{d}^{\mathrm{T}}(\omega)}$,

where the transverse coefficients $(5.16)$ have to be inserted into $I_{a}^{\mathrm{T}}(\omega)$. Because of the divergence of the static susceptibility in the whole ordered phase, the transverse modes become hydrodynamic; i.e., their frequency depends on wave number as $\sqrt{(c / I)} q$. According to (5.20), these modes become overdamped for small $q$ and are diffusive. Parenthetically, we note that for $v=0$ the free-energy functional, $(2.4 \mathrm{a})$ is $\mathrm{O}(M)$ symmetric. In this

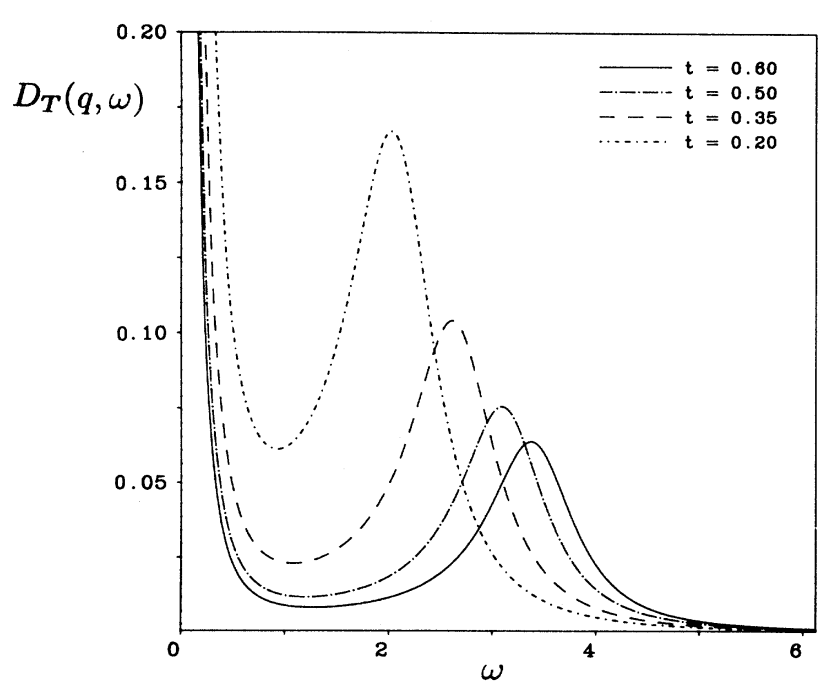

FIG. 14. Transverse phonon-phonon correlation function $D_{\mathrm{T}}(\mathbf{q}, \omega)$ for an isotropic system $(v=0)$ below $T_{c}(n)$, for several temperatures (see Figs. 6 and 12). 
case a different type of dynamics is conceivable, namely, equations of motion supplemented by the conservation law of angular momentum. For such dynamics the transverse modes would have linear dispersion and quadratic (diffusive) damping. ${ }^{44}$ Returning to (5.20), a dynamical central peak thus persists below $T_{c}(n)$ in the transverse dynamical correlation function (Fig. 14), provided that there are no cubic terms in the free-energy expansion (2.4a).

In reality, terms of cubic symmetry are present $(v \neq 0)$, and therefore it is interesting to study their influence on the transverse correlation function. Figure 15 shows
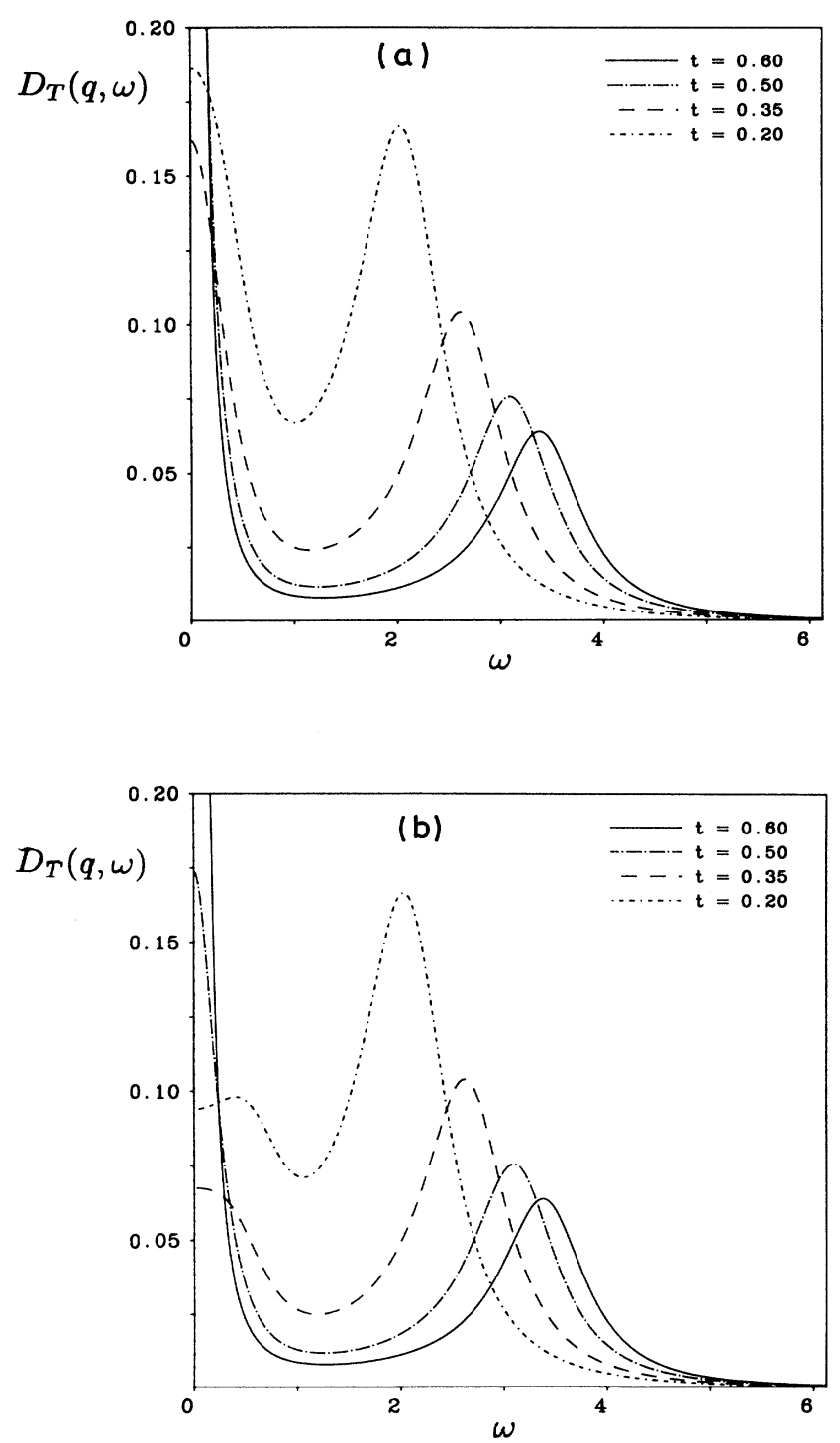

FIG. 15. Transverse phonon-phonon correlation function $D_{\mathrm{T}}(\mathbf{q}, \omega)$ below $T_{c}(n)$, for several temperatures, taking into account cubic terms $[y=-v /(b-v)>0]$. (a) $y=0.06$; (b) $y=0.1$. The graph for $y=0.01$ is indistinguishable from Fig. 14 , corresponding to $v=0$.

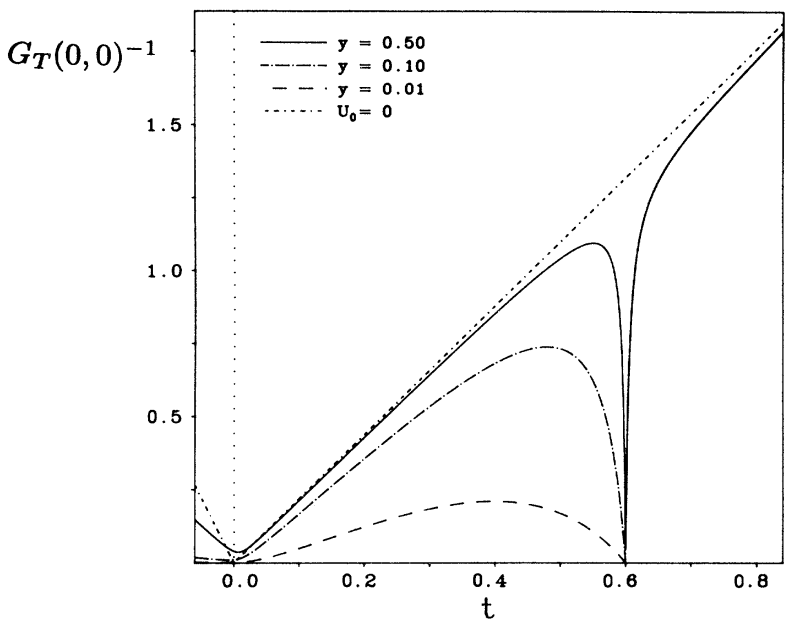

FIG. 16. Inverse transverse static susceptibility $G_{\mathrm{T}}^{-1}(0,0)$ for several values of $y=-v /(b-v)$.

several Plots of $D_{\mathrm{T}}(\mathbf{q}, \omega)$ for $q=2.8 \times 10^{6} \mathrm{~cm}^{-1}$ and for different values of the ratio $y=-v /(b-v), v<0$. For low values of this ratio, $y$, the coexistence singularity, remains relevant in a fairly large temperature range and a dynamical central peak persists in the transverse correlation function. For $y=0.01$ the graph is indistinguishable from $y=0$ shown in Fig. 14. These features are

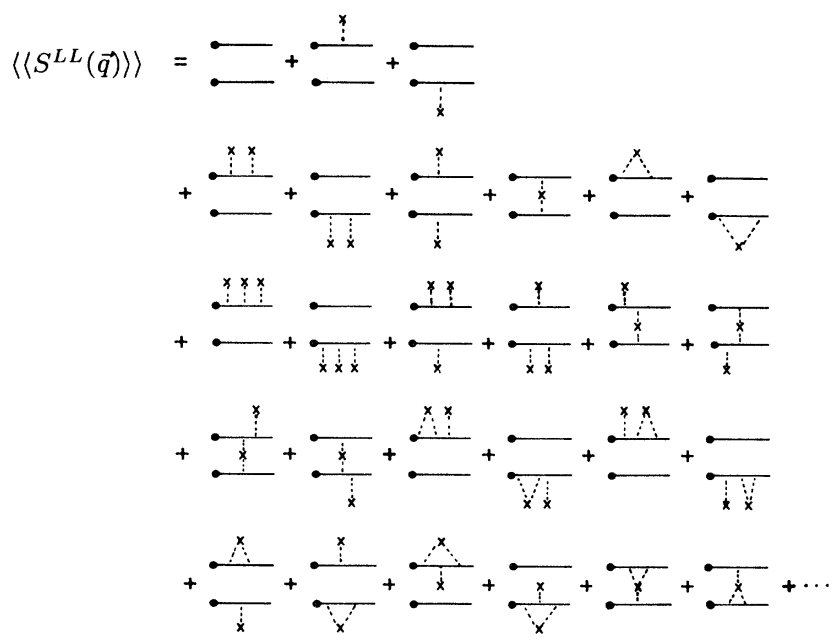

(a)

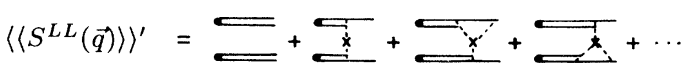

(b)

FIG. 17. (a) Diagrams for $\left\langle\left\langle S^{\mathrm{LL}}(\mathbf{q})\right\rangle\right\rangle$, the static contribution to the dynamical structure factor (Huang scattering). (b) Summation of diagrams from (a) according to single-site approximation. 
confirmed by the temperature dependence of the inverse static transverse susceptibility $G_{\mathrm{T}}^{-1}(0,0)$ shown in Fig. 16 for different cubic anisotropy coefficients. We remark that $\mathrm{SrTiO}_{3}$ displays a considerable anisotropy, with a value of $y \approx 0.23$, while the anisotropy is less in the case of $\mathrm{LaAlO}_{3}$.

\section{Elastic part of the structure function}

Now we turn to the elastic contribution to the scattering cross section of Eq. (2.11). The part related to the static order-parameter fluctuation is given by Eq. (2.12) and has to be averaged over the defect configurations:

$$
\left\langle\left\langle S^{\alpha \beta}(\mathbf{q})\right\rangle\right\rangle=\left\langle\left\langle\frac{1}{N} \sum_{1 \leq i, j \leq N} e^{-i \mathbf{q} \cdot\left(\mathbf{a}_{i}-\mathbf{a}_{j}\right)} \bar{\psi}_{i}^{\mathrm{L}} \bar{\psi}_{j}^{\mathrm{L}}\right\rangle\right\rangle \delta^{\alpha \mathrm{L}} \delta^{\beta \mathrm{L}} .
$$

Note that this defect average does not factorize into $\left\langle\left\langle\bar{\psi}^{\mathrm{L}}\right\rangle\right\rangle\left\langle\left\langle\bar{\psi}^{\mathrm{L}}\right\rangle\right\rangle$.

We evaluate the quantity $\left\langle\left\langle S^{\mathrm{LL}}(\mathbf{q})\right\rangle\right\rangle$ by inserting Eq. (5.5) for $\psi_{i}^{\mathrm{L}}$ into (5.21). After a Fourier transformation we find

$$
S^{\mathrm{LL}}(\mathbf{q})=h^{2}\left[\widetilde{G}_{0}(\mathbf{0})\right]^{2} \delta_{\mathbf{q}, 0}+h\left[\widetilde{G}_{0}(\mathbf{0})\right]^{2} \sum_{\mathbf{k}} \widetilde{\Phi}_{0 \mathbf{k}} \bar{\psi}_{\mathbf{k}}^{\mathrm{L}} \delta_{\mathbf{q}, 0}+\widetilde{G}_{0}(\mathbf{q}) \sum_{\mathbf{k}} \widetilde{\Phi}_{\mathbf{q} \mathbf{k}} \bar{\psi}_{\mathbf{k}}^{\mathrm{L}} \bar{\psi}_{\mathbf{q}}^{\mathrm{L}}
$$

Now we can use Eq. (5.8) for $\bar{\psi}_{\mathbf{q}}^{\mathrm{L}}$ and thus iteratively determine $\left\langle\left\langle S^{\mathrm{LL}}(\mathbf{q})\right\rangle\right\rangle$ by performing the configurational average over the defect positions (4.12). Again, a diagrammatic representation for the corresponding perturbation theory can be derived [see Fig. 17(a)] consisting of the same basic ingredients as for the order parameter. In Fig. 17(b) the diagrammatic summation of the contributions according to single-site approximation is illustrated. Note that the "disconnected" diagrams are just proportional to $N \sum_{\mathrm{g}} \delta_{\mathrm{q}, \mathrm{g}}\left\langle\left\langle\bar{\psi}^{\mathrm{L}}\right\rangle\right\rangle^{2}$, while the "connected" graphs yield a $q$-dependent contribution. The result is

$$
\begin{aligned}
\left\langle\left\langle S^{\mathrm{LL}}(\mathbf{q})\right\rangle\right\rangle^{\prime} & =\left\langle\left\langle\bar{\psi}^{\mathrm{L}}\right\rangle\right\rangle^{2} N \sum_{\mathbf{g}} \delta_{\mathbf{q}, \mathbf{g}}+n \widetilde{\lambda}^{2}\left\langle\left\langle\bar{\psi}^{\mathrm{L}}\right\rangle\right\rangle^{2}\left[\widetilde{G}_{0}(\mathbf{q})\right]^{2}\left[1+2 \frac{\widetilde{\lambda}}{N} \sum_{\mathbf{k}} \widetilde{G}_{0}(\mathbf{k})+3\left[\frac{\widetilde{\lambda}}{N} \sum_{\mathbf{k}} \widetilde{G}_{0}(\mathbf{k})\right]^{2}+\cdots\right] \\
& =\left\langle\left\langle\bar{\psi}^{\mathrm{L}}\right\rangle\right\rangle^{2}\left[N \sum_{\mathbf{g}} \delta_{\mathbf{q}, \mathbf{g}}+n \widetilde{\lambda}^{2} \frac{\left[\widetilde{\boldsymbol{G}}_{0}(\mathbf{q})\right]^{2}}{\left[1-(\tilde{\lambda} / N) \sum_{\mathbf{k}} \widetilde{\boldsymbol{G}}_{0}(\mathbf{k})\right]^{2}}\right] \\
& =\left\langle\left\langle\bar{\psi}^{\mathrm{L}}\right\rangle\right\rangle^{2}\left(N \sum_{\mathbf{g}} \delta_{\mathbf{q}, \mathbf{g}}+\frac{1}{n} \frac{\left[\widetilde{G}_{0}(\mathbf{q})\right]^{2}}{\left[\widetilde{G}_{0}(\mathbf{0})\right]^{2}}\right],
\end{aligned}
$$

where in the final step Eq. (5.11) for the order parameter in zero external field was used. Here $\widetilde{G}_{0}(\mathbf{q})$ has been defined in Eq. (5.6a) and $n$ is the defect concentration.

Thus, for zone-boundary soft modes, we find, as is expected, a contribution to the elastic-scattering cross section proportional to $\sin ^{2}(\mathbf{k} \cdot\langle\langle\bar{\psi}\rangle\rangle) \approx(\mathbf{k} \cdot\langle\langle\bar{\psi}\rangle\rangle)^{2}$, i.e., the square of the averaged order parameter (5.3) [see Eq. (2.11)]. This is the Bragg scattering due to the appearance of the order parameter $\left\langle\left\langle\bar{\psi}^{\mathrm{L}}\right\rangle\right\rangle$ below $T_{c}(n)$. For antiferrodistortive transitions these peaks appear at the reciprocal-lattice points $\mathbf{q}_{0}+\mathbf{g}$ of the low-temperature phase. However, there is an additional elastic central-peak contribution with finite width in momentum space stemming from the underlying inhomogeneous structure. Its scattering intensity is proportional to $\left[\widetilde{G}_{0}(q)\right]^{2}$, and the $q$ width is given by the corresponding "correlation length" $\left(a+b A^{2}\right) / c$, which is not divergent at $T_{c}^{0}$ because of the finite order parameter $\left\langle\left\langle\bar{\psi}^{\mathrm{L}}\right\rangle\right\rangle$. In Fig. 18(a) we display the elastic-scattering contribution $\left\langle\left\langle S_{C}^{\mathrm{LL}}(\mathbf{q})\right\rangle\right\rangle^{\prime}$ [second term in Eq. (5.22b)] for several wave numbers as a function of the temperature. For small concentration this intensity is almost zero for $T \gg T_{c}^{0}$, but increases strongly for $T \rightarrow T_{c}^{0}$. In order to emphasize the drastic increase of the Huang scattering, we note that in Fig. 18(a) its value at $T_{c}^{0}$ is four orders of magnitude higher than at $T=1.5 T_{c}^{0}$. In Fig. 18(b) the quantity $\widetilde{a} / b$, which is proportional to the $q$ width, is shown. The $q$ width narrows when $T_{c}^{0}$ is approached, but never becomes zero because of the underlying inhomogeneous order parameter.

At this point we sum up the dynamic and static contributions contained in the averaged scattering cross section (2.11). The complete dynamical structure factor

$$
\begin{aligned}
\langle\langle S(\mathbf{k}, \omega)\rangle= & 2 \pi \mid N \sum_{\mathbf{g}} \delta_{\mathbf{k}, \mathbf{g}} \cos ^{2}\left(k^{\mathrm{L}}\left\langle\left\langle\bar{\psi}^{\mathrm{L}}\right\rangle\right\rangle\right)+N \sum_{\mathbf{g}} \delta_{\mathbf{k}-\mathbf{q}_{0}, \mathbf{g}} \sin ^{2}\left(k^{\mathrm{L}}\left\langle\left\langle\bar{\psi}^{\mathrm{L}}\right\rangle\right\rangle\right)+\left(k^{\mathrm{L}}\right)^{2}\left\langle\left\langle\bar{\psi}^{\mathrm{L}}\right\rangle\right\rangle^{2} \frac{1}{n} \frac{\left[\widetilde{\boldsymbol{G}}_{0}(\mathbf{k})\right]^{2}}{\left[\widetilde{\boldsymbol{G}}_{0}(\mathbf{0})\right]^{2}} \sin ^{2}\left(k^{\mathrm{L}}\left\langle\left\langle\bar{\psi}^{\mathrm{L}}\right\rangle\right\rangle\right) \\
& \left.+\left(k^{\mathrm{L}}\right)^{2}\left\langle\left\langle\bar{\psi}^{\mathrm{L}}\right\rangle\right\rangle^{2} \frac{1}{n} \frac{\left[\widetilde{\boldsymbol{G}}_{0}\left(\mathbf{k}-\mathbf{q}_{0}\right)\right]^{2}}{\left[\widetilde{\boldsymbol{G}}_{0}(\mathbf{0})\right]^{2}} \cos ^{2}\left(k^{\mathrm{L}}\left\langle\left\langle\bar{\psi}^{\mathrm{L}}\right\rangle\right\rangle\right)\right] e^{-2 W} \delta(\omega) \\
+ & \left(\sum_{\alpha}\left(k^{\alpha}\right)^{2} D^{\alpha \alpha}(\mathbf{k}, \omega) \sin ^{2}\left(k^{\mathrm{L}}\left\langle\left\langle\bar{\psi}^{\mathrm{L}}\right\rangle\right\rangle\right)+\sum_{\alpha}\left(k^{\alpha}\right)^{2} D^{\alpha \alpha}\left(\mathbf{k}-\mathbf{q}_{0}, \omega\right) \cos ^{2}\left(k^{\mathrm{L}}\left\langle\left\langle\bar{\psi}^{\mathrm{L}}\right\rangle\right\rangle\right)\right] e^{-2 W}
\end{aligned}
$$


consists of (i) the standard Bragg contribution (first term), (ii) the Bragg contribution due to the order parameter (second term), (iii) the elastic central peaks with finite $q$ width (third and fourth term), and (iv) the dynamical contributions (5.15) and (5.20), which under the conditions mentioned in Sec. V B can also contain a dynamical central peak. None of these central peaks diverge at the pure transition temperature $T_{c}^{0}$. From (2.12) and (2.13) it follows that $\left\langle\left\langle S_{C}^{\mathrm{LL}}(\mathbf{k}+\mathbf{g})\right\rangle\right\rangle=\left\langle\left\langle S_{C}^{\mathrm{LL}}(\mathbf{k})\right\rangle\right\rangle$ and $D^{\alpha \alpha}(\mathbf{k}+\mathbf{g}, \omega)=D^{\alpha \alpha}(\mathbf{k}, \omega)$. The detailed predictions of this theory should permit a quantitative comparison with experiments and in turn shed some light onto the underlying mechanism of the central-peak phenomenon.
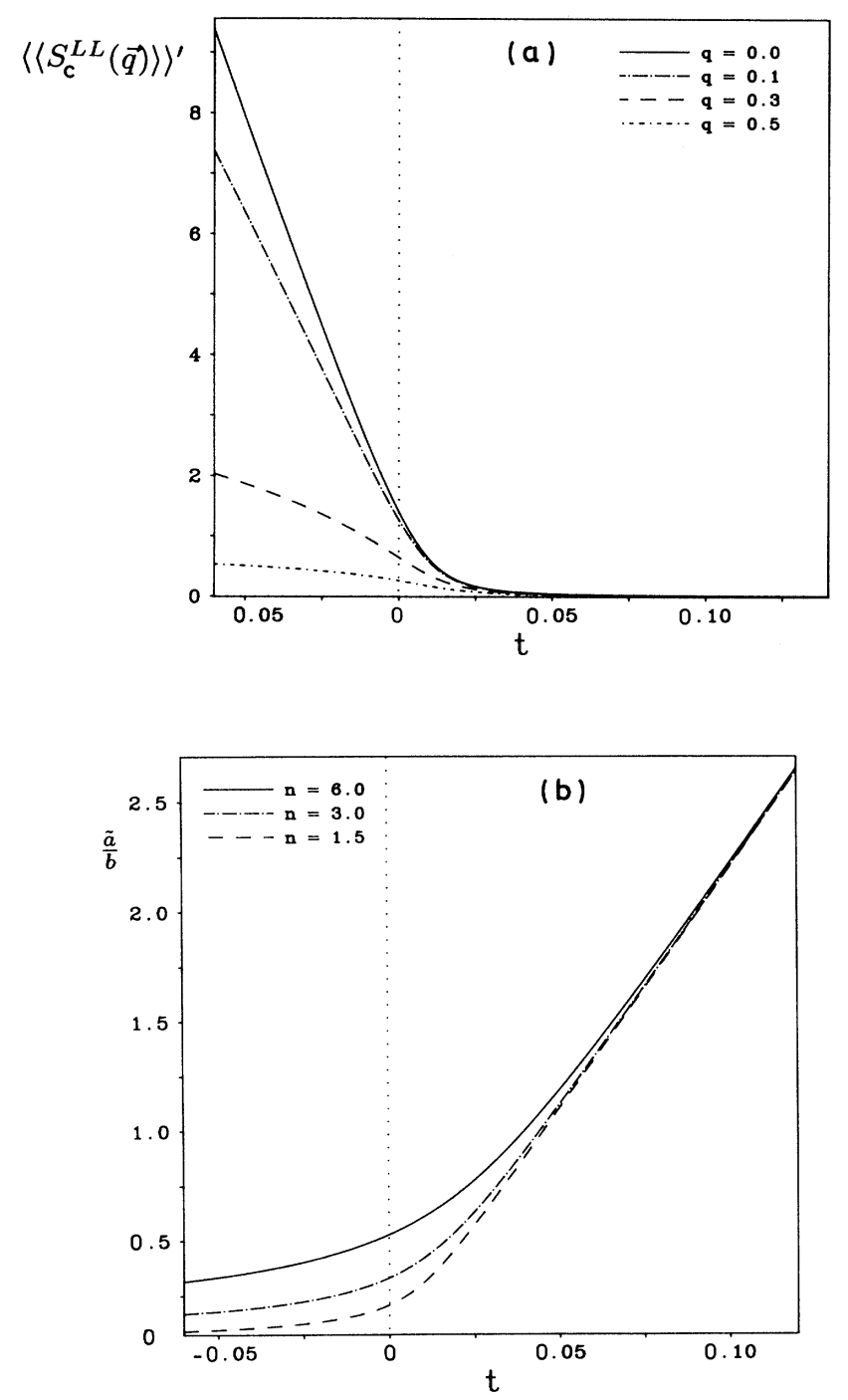

FIG. 18. (a) Intensity of the central peak contribution with finite $q$ width [second term in Eq. (5.22b)] vs the temperature for several wave numbers $q$ ( $q$ in units $2.8 \times 10^{6} \mathrm{~cm}^{-1}$ ). (b) $q$ width of the elastic central peak [second term of Eq. $(5.22 b)] \propto \widetilde{a} / b$ as a function of the temperature.

\section{DISCUSSION OF THE RESULTS AND OUTLOOK}

In this final section, we summarize our results, discuss possible applications to experiments, and compare with other theories.

In summary, we have considered the influence of randomly distributed defects which locally increase the transition temperature at distortive structural phase transformations. The defects modify the quadratic term of the free-energy functional, and the internal symmetry is not broken. As a consequence, below the continuum of optic-phonon states there appears a band of localized modes. In one dimension this impurity band will exist for any value of the defect strength, while in three dimensions the strength of the $T_{c}$-increasing (attractive) potential has to exceed a certain threshold. This impurity band softens above the bulk transition temperature of the pure system, at a temperature $T_{c}(n)$. Below $T_{c}(n)$ order sets in. Close to $T_{c}(n)$ the order parameter is of appreciable magnitude only in the immediate vicinity of the defects, more and more spreading throughout the entire crystal with decreasing temperature. The impurityaveraged order parameter has been computed in Sec. V A. Its temperature dependence is a function of the impurity concentration $n$. For low concentration the order parameter remains very small, of $O(n)$, in the whole temperature interval $T_{c}^{0}<T<T_{c}(n)$ and builds up to detectable values only near $T_{c}^{0}$. Compared with the order parameter of the pure system, which is of the form $\bar{\psi}^{\mathrm{L}} \propto\left(T_{c}^{0}-T\right)^{\beta}\left(\beta=\frac{1}{2}\right.$ in the Ginzburg-Landau approximation), it looks as if this order parameter curve were rounded, as is sometimes observed in experiments.

In scattering experiments there appears a Bragg intensity due to this order parameter for temperatures $T \leq T_{c}(n)$. Furthermore, because of the random and inhomogeneous static configuration, the elastic-scattering intensity is not just given by the square of the order parameter, but contains an additional contribution which has a finite $q$ width-as observed in many of the centralpeak experiments.

In the dynamics we find as a precursor of the impurity-induced condensation a dynamical central peak for $T$ very closely above $T_{c}(n)$, becoming very narrow when the transition is approached, which stems from the overdamped impurity band. In one-component systems this peak broadens again for $T<T_{c}(n)$ and finally disappears into the continuum of extended optic-phonon states. For an $\boldsymbol{M}$-dimensional order parameter, the longitudinal component (parallel to the direction of the order parameter) behaves in exactly the same manner. However, the transverse components may contain a dynamical central peak. This is a function of the anisotropy of the nonlinear coupling constants. For a Heisenberg system (displaying continuous rotational invariance), a central peak persists in the ordered phase because of the Goldstone theorem. For weak anisotropy $(y=|v| /$ $(b-v) \leq 0.05$, say), the central mode can be found in a considerable temperature range. For higher anisotropy the transverse dynamical central peak disappears similarly to the longitudinal one. Thus we have found a remarkably rich behavior, which probably can accommodate 
many of the previous experimental results.

As compared to the single-defect treatments which underestimate cooperative effects, the scope of the present theory and the theoretical picture resulting therefrom have changed rather drastically. Instead of a bound state, there is a band of impurity states. Instead of independent clusters with a slow relaxation rate, a weak ordered state builds up. This ordered state can be characterized by a finite static order parameter. Furthermore, it gives rise to a static central peak (with zero frequency width) with a finite $q$ width. However, we have to be aware of the fact that Ginzburg-Landau theory exaggerates collective behavior by neglecting fluctuations.

At this point we would like to add some comments on the limits and range of validity of our theory. Strictly speaking, it must break down for very small defect concentrations, because for small concentration, nonoverlapping local moments will be formed at $T_{c}^{l}$, which will order only at a temperature lower than $T_{c}^{l}$. The mean-field approach takes into account the lowest minimum of the free-energy functional only and implies that order always sets in with the formation of the moments. For small concentrations the energy gap between different local minima of the free-energy functional is exponentially small. One can easily estimate the temperature at which the moments order, by equating this temperature with the energy gap. The limiting effects become less important for (i) extended defects, i.e., a net of grain boundaries with increased transition temperature, and (ii) phase transitions at very low temperatures.

The theory may be of practical use for the interpretation of experiments even in the concentration range, where, strictly speaking, there is no finite order parameter in the thermodynamic sense. Then the local moments are not ordered, but fluctuate with a reorientation rate, which is very small due to the high energy barrier between equivalent states. ${ }^{30,31}$ Both the Bragg peak and Huang scattering of Eq. (5.24) will acquire a finite frequency width. The dynamic term will be essentially unchanged for frequencies large compared to the reorientation rate. For temperatures close to $T_{c}^{0}$, the frequency widths will be experimentally unobservable, implying, for instance, that the experimental "order parameter" will appear in a shape similar to Fig. 10.

In this paper we have concentrated mainly on the overall physical picture, in order to understand the mechanisms of the central-peak phenomenon. The GinzburgLandau parameters used (Table I) are comparable to those for typical structural transitions, but no specific attempt has been made to fit a particular substance. Clearly, the theory can also be implemented into detailed lattice-dynamical computations, e.g., the shell model, etc.

We believe that the mechanism presented here will have many applications to real systems. Of course, other mechanisms are not ruled out, however. For instance, slowly relaxing defects give a dynamical central peak; static random fields produce a static central peak, and so forth. For summaries of the various conceivable mechanisms, we refer the reader to Refs. 11,30 , and 31 . We would like to briefly contrast our results with two different models, namely, (i) Huang scattering from static defects which couple linearly to the order parameter and (ii) the coupling of a relaxational mode to the order parameter. The standard treatment of Huang scattering is based on a linear superposition of the displacements emerging from the individual defects. This gives rise to a scattering intensity proportional to the square of the static susceptibility of the pure system. Also, in case (ii), the strength of the (now dynamical) central peak is proportional to the divergent static susceptibility.

Obviously, the present theory has applications to other phase transitions as well. For instance, elastic phase transitions can be studied quite analogously. ${ }^{45}$ But even beyond the field of structural phase transformations, defects which locally increase the transition temperature may be of importance (superconductors, magnetic systems). Concerning the static behavior, our results and conclusions can be taken over without changes, while the dynamics are, of course, different. It is quite clear, however, how to apply this theory also to the relaxational or diffusive dynamics of superconductors or magnets. The dynamical precursor then is an extra, even more narrow relaxational or diffusive mode, respectively.

Concerning the experimental verification of our theory, we have discussed primarily scattering experiments, because of their direct relation to the wave-number- and frequency-dependent density-density correlation functions exhibiting the underlying excitations of the system. Other experimental probes, such as EPR and ultrasonic attenuation, show anomalies as well. These can also be studied on the basis of our theory.

\section{ACKNOWLEDGMENTS}

This work has been supported by the German Federal Ministry of Research and Technology (BMFT) under Contract No. 03-SC2TUM-0.

\section{APPENDIX A: NEUTRON-SCATTERING CROSS SECTION}

In this appendix we indicate how the scattering law from Sec. II B is generalized to lattices with a basis and realistic polarization vectors. The essential dependence on the order parameter and the correlation function remains unchanged; only the geometrical prefactors are modified.

The lattice positions are given by

$$
\mathbf{a}_{\mathbf{n}, s}=\mathbf{a}_{\mathbf{n}}+\mathbf{b}_{s}
$$

where $\mathbf{a}_{\mathbf{n}}$ is a lattice vector and $\mathbf{b}_{s}$ is the position of atom $s$ within the unit cell. The actual positions of the atoms are represented by the normal-mode expansion

$$
\begin{aligned}
\mathbf{x}_{\mathbf{n}, s} & =\mathbf{a}_{\mathbf{n}, s}+\phi_{\mathbf{n}, s} \\
& =\mathbf{a}_{\mathbf{n}, s}+\frac{1}{\sqrt{N M_{s}}} \sum_{\mathbf{k}, \alpha} e^{i \mathbf{k} \cdot \mathbf{a}_{\mathbf{n}}} \boldsymbol{\epsilon}_{s}(\mathbf{k}, \alpha) Q_{\mathbf{k}, \alpha},
\end{aligned}
$$

where $Q_{\mathbf{k}, \alpha}$ are the normal modes, $\boldsymbol{\epsilon}_{s}(\mathbf{k}, \alpha)$ the polarization vectors, and $M_{s}$ the mass of atom $s$. A certain subset of these $Q_{\mathrm{k}, \alpha}$ coincides with the critical normal modes studied in this paper; i.e., 


$$
Q_{\mathbf{k}, \alpha}=\psi_{\mathbf{k}-\mathbf{q}_{0}}^{\alpha}
$$

Restricting ourselves just to the critical degrees of freedom, we have

$$
\phi_{\mathbf{n}, s}=\frac{1}{\sqrt{N M_{s}}} \sum_{\mathbf{k}, \alpha} e^{i \mathbf{k} \cdot \mathbf{a}_{\mathbf{n}}} \boldsymbol{\epsilon}_{s}(\mathbf{k}, \alpha) \psi_{\mathbf{k}-\mathbf{q}_{0}}^{\alpha} .
$$

Here $\psi_{q}^{\alpha}$ is governed by the free-energy expansion and the equations of motion of Sec. II. Now $\phi_{n, s}$ can be written as

$$
\boldsymbol{\phi}_{\mathbf{n}, s}=\left\langle\left\langle\overline{\boldsymbol{\phi}}_{\mathbf{n}, s}\right\rangle\right)+\left(\overline{\boldsymbol{\phi}}_{\mathbf{n}, s}-\left\langle\left\langle\overline{\boldsymbol{\phi}}_{\mathbf{n}, s}\right\rangle\right\rangle\right)+\mathbf{u}_{\mathbf{n}, s},
$$

where

$$
\overline{\boldsymbol{\phi}}_{\mathrm{n}, s}=\left\langle\phi_{\mathrm{n}, s}\right\rangle
$$

and

$$
\begin{aligned}
\left\langle\left\langle\bar{\phi}_{\mathbf{n}, s}\right\rangle\right\rangle & =\frac{1}{\sqrt{N M_{s}}} \sum_{\mathbf{k}, \alpha} e^{i \mathbf{k} \cdot \mathbf{a}_{\mathbf{n}}} \boldsymbol{\epsilon}_{s}(\mathbf{k}, \alpha)\left\langle\left\langle\bar{\psi}^{\alpha}\right\rangle\right\rangle \delta_{\mathbf{k}, \mathbf{q}_{0}} \\
& =e^{i \mathbf{q}_{0} \cdot \mathbf{a}_{\mathbf{n}}} \delta_{s}
\end{aligned}
$$

with

$$
\delta_{s}=\frac{1}{\sqrt{N M_{s}}} \sum_{\alpha} \epsilon_{s}\left(\mathbf{q}_{0}, \alpha\right)\left\langle\left\langle\bar{\psi}^{\alpha}\right\rangle\right\rangle .
$$

The phonon displacements are abbreviated by $\mathbf{u}_{\mathbf{n}, s}$.

The scattering length of atom $s$ is denoted by $f_{s}$. Assuming for the sake of simplicity that the defects do not modify the scattering legnths, the coherent neutronscattering cross section is proportional to

$$
\langle\langle S(\mathbf{k}, \omega)\rangle\rangle=\left\langle\left\langle\int e^{i \omega t}\left\langle\sum_{\mathbf{n}, s} \bar{f}_{s} e^{-i \mathbf{k} \cdot \mathbf{x}_{\mathbf{n}, s}(t)} \sum_{\mathbf{n}^{\prime}, s^{\prime}} \bar{f}_{s^{\prime}}^{*} e^{i \mathbf{k} \cdot \mathbf{x}_{\mathbf{n}^{\prime}, s^{\prime}}(0)}\right\rangle d t\right\rangle .\right.
$$

The averaged dynamical structure factor of Eq. (A9) can be evaluated by following the steps of Eqs. (2.8)-(2.11) of Sec. II.

For a zone-center soft mode $\left(\mathbf{q}_{0}=0\right)$, we find

$$
\begin{aligned}
\langle\langle S(\mathbf{k}, \omega)\rangle\rangle=\sum_{s, s^{\prime}} & \bar{f}_{s} \bar{f}_{s^{\prime}}^{*} e^{-i \mathbf{k} \cdot\left(\mathbf{b}_{s}+\delta_{s}-\mathbf{b}_{s^{\prime}}-\delta_{s^{\prime}}\right)} e^{-W_{s}-W_{s^{\prime}}} \\
\times & {\left[2 \pi \mid N \sum_{\mathbf{g}} \delta_{\mathbf{k}, \mathbf{g}}+\frac{1}{\sqrt{\boldsymbol{M}_{s} \boldsymbol{M}_{s^{\prime}}}} \sum_{\alpha}\left[\mathbf{k} \cdot \boldsymbol{\epsilon}_{s}(\mathbf{k}, \alpha)\right]\left[\mathbf{k} \cdot \boldsymbol{\epsilon}_{s^{\prime}}^{*}(\mathbf{k}, \alpha)\right]\left\langle\left\langle S_{C}^{\alpha \alpha}(\mathbf{k})\right\rangle\right\rangle \delta \delta\right) } \\
& \left.\quad+\frac{1}{\sqrt{\boldsymbol{M}_{s} \boldsymbol{M}_{s^{\prime}}}} \sum_{\alpha}\left[\mathbf{k} \cdot \boldsymbol{\epsilon}_{s}(\mathbf{k}, \alpha)\right]\left[\mathbf{k} \cdot \boldsymbol{\epsilon}_{s^{\prime}}^{*}(\mathbf{k}, \alpha)\right] D^{\alpha \alpha}(\mathbf{k}, \omega)\right],
\end{aligned}
$$

where the Debye-Waller factor is given by

$$
W_{s}=\frac{1}{2}\left\langle\left\langle\left[\mathbf{k} \cdot\left(\overline{\boldsymbol{\phi}}_{\mathbf{n}, s}-\left\langle\left\langle\overline{\boldsymbol{\phi}}_{\mathbf{n}, s}\right\rangle\right\rangle\right)\right]^{2}\right\rangle\right\rangle+\frac{1}{2}\left\langle\left\langle\left\langle\left(\mathbf{k} \cdot \mathbf{u}_{\mathbf{n}, s}\right)^{2}\right\rangle\right\rangle\right\rangle .
$$

For a zone-boundary soft mode $\left(\mathbf{q}_{0} \neq 0\right.$ and $\left.e^{2 i \mathbf{q}_{0} \cdot \mathbf{a}_{\mathbf{n}}}=1\right)$, the result is

$$
\begin{aligned}
& \langle\langle S(\mathbf{k}, \omega)\rangle\rangle=\sum_{s, s^{\prime}} \bar{f}_{s} \bar{f}_{s^{\prime}}^{*} e^{-i \mathbf{k} \cdot\left(\mathbf{b}_{s}-\mathbf{b}_{s^{\prime}}\right)} e^{-W_{s}-W_{s^{\prime}}} \\
& \times\left[2 \pi \left(N \sum_{\mathbf{g}} \delta_{\mathbf{k}, \mathrm{g}} \cos \left(\mathbf{k} \cdot \boldsymbol{\delta}_{s}\right) \cos \left(\mathbf{k} \cdot \boldsymbol{\delta}_{s^{\prime}}\right)+N \sum_{\mathrm{g}} \delta_{\mathbf{k}-\mathbf{q}_{0}, \mathrm{~g}} \sin \left(\mathbf{k} \cdot \boldsymbol{\delta}_{s}\right) \sin \left(\mathbf{k} \cdot \boldsymbol{\delta}_{s^{\prime}}\right)\right.\right. \\
& +\frac{1}{\sqrt{M_{s} M_{s^{\prime}}}} \sum_{\alpha}\left[\mathbf{k} \cdot \boldsymbol{\epsilon}_{s}\left(\mathbf{k}+\mathbf{q}_{0}, \alpha\right)\right]\left[\mathbf{k} \cdot \boldsymbol{\epsilon}_{s^{\prime}}^{*}\left(\mathbf{k}+\mathbf{q}_{0}, \alpha\right)\right]\left\langle\left\langle S_{c}^{\alpha \alpha}(\mathbf{k})\right\rangle\right\rangle \sin \left(\mathbf{k} \cdot \boldsymbol{\delta}_{s}\right) \sin \left(\mathbf{k} \cdot \boldsymbol{\delta}_{s^{\prime}}\right) \\
& \left.+\frac{1}{\sqrt{M_{s} M_{s^{\prime}}}} \sum_{\alpha}\left[\mathbf{k} \cdot \boldsymbol{\epsilon}_{s}(\mathbf{k}, \alpha)\right]\left[\mathbf{k} \cdot \boldsymbol{\epsilon}_{s^{\prime}}^{*}(\mathbf{k}, \alpha)\right]\left\langle\left\langle S_{C}^{\alpha \alpha}\left(\mathbf{k}-\mathbf{q}_{0}\right)\right\rangle\right\rangle \cos \left(\mathbf{k} \cdot \boldsymbol{\delta}_{s}\right) \cos \left(\mathbf{k} \cdot \boldsymbol{\delta}_{s^{\prime}}\right)\right] \delta(\omega) \\
& +\frac{1}{\sqrt{M_{s} M_{s^{\prime}}}} \sum_{\alpha}\left[\mathbf{k} \cdot \boldsymbol{\epsilon}_{s}\left(\mathbf{k}+\mathbf{q}_{0}, \alpha\right)\right]\left[\mathbf{k} \cdot \boldsymbol{\epsilon}_{s^{\prime}}^{*}\left(\mathbf{k}+\mathbf{q}_{0}, \alpha\right)\right] D^{\alpha \alpha}(\mathbf{k}, \omega) \sin \left(\mathbf{k} \cdot \boldsymbol{\delta}_{s}\right) \sin \left(\mathbf{k} \cdot \boldsymbol{\delta}_{s^{\prime}}\right) \\
& \left.+\frac{1}{\sqrt{M_{s} M_{s^{\prime}}}} \sum_{\alpha}\left[\mathbf{k} \cdot \boldsymbol{\epsilon}_{s}(\mathbf{k}, \alpha)\right]\left[\mathbf{k} \cdot \boldsymbol{\epsilon}_{s^{\prime}}^{*}(\mathbf{k}, \alpha)\right] D^{\alpha \alpha}\left(\mathbf{k}-\mathbf{q}_{0}, \omega\right) \cos \left(\mathbf{k} \cdot \boldsymbol{\delta}_{s}\right) \cos \left(\mathbf{k} \cdot \boldsymbol{\delta}_{s^{\prime}}\right)\right] \text {. }
\end{aligned}
$$

The correlation functions $\left\langle\left\langle S_{C}^{\alpha \alpha}(\mathbf{q})\right\rangle\right\rangle$ and $D^{\alpha \alpha}(\mathbf{q}, \omega)$ describe Huang scattering and one-phonon scattering, respectively, and have been evaluated for small arguments in Secs. IV and V. We also note that $\boldsymbol{\epsilon}_{s}(\mathbf{k}+\mathbf{g}, \alpha)=\boldsymbol{\epsilon}_{s}(\mathbf{k}, \alpha)$, $\left\langle\left\langle S_{C}^{\alpha \alpha}(\mathbf{k}+\mathbf{g})\right\rangle\right\rangle=\left\langle\left\langle S_{C}^{\alpha \alpha}(\mathbf{k})\right\rangle\right\rangle$, and $D^{\alpha \alpha}(\mathbf{k}+\mathbf{g}, \omega)=D^{\alpha \alpha}(\mathbf{k}, \omega)$. 


\section{APPENDIX B: DEFECT-AVERAGED RESPONSE FUNCTION, A SECOND DERIVATION}

We present here a second derivation of the phonon-phonon response function of a random multidefect system [Eqs. (4.16) and (4.17)] based on the exact single-defect susceptibility and the defect average thereof.

We split the Hamiltonian for a single-defect system [compare (3.1), for instance] into a "free" part $H_{0}$ and the singledefect potential $V\left(\mathbf{x}_{D}\right)$ according to

$$
H=H_{0}+V\left(\mathbf{x}_{D}\right) \text {, }
$$

and define its eigenfunctions and eigenvalues by the equation

$$
H f_{\mathbf{q}}(\mathbf{k}) e^{-i \mathbf{k} \cdot \mathbf{x}_{D}}=\varepsilon_{\mathbf{q}} f_{\mathbf{q}}(\mathbf{k}) e^{-i \mathbf{k} \cdot \mathbf{x}_{D}} .
$$

The "full" defect-dependent dynamical response function is then given by standard formula

$$
G\left(\mathbf{k}, \mathbf{k}^{\prime} ; \mathbf{x}_{D}\right)=\sum_{\mathbf{q}} \frac{f_{\mathrm{q}}(\mathbf{k}) f_{\mathrm{q}}^{*}\left(\mathbf{k}^{\prime}\right)}{\omega^{2}-\varepsilon_{\mathrm{q}}} e^{-i\left(\mathbf{k}-\mathbf{k}^{\prime}\right) \cdot \mathbf{x}_{D}},
$$

whereas the free propagator is translationally invariant and reads

$$
G_{0}\left(\mathbf{k}, \mathbf{k}^{\prime}\right)=\delta_{\mathbf{k} \mathbf{k}^{\prime}} \frac{1}{\omega^{2}-\omega_{\mathbf{k}}^{2}},
$$

where $\omega_{\mathbf{k}}^{2}$ denote the eigenvalues of $H_{0}$.

We now turn to the case of $N_{D}$ widely separated defects. The response function can be represented by

$$
G\left(\mathbf{k}, \mathbf{k}^{\prime} ; \mathbf{x}_{D_{1}}, \ldots, \mathbf{x}_{D_{N_{D}}}\right)=G_{0}\left(\mathbf{k}, \mathbf{k}^{\prime}\right)+\sum_{i_{D}=1}^{N_{D}}\left[G\left(\mathbf{k}, \mathbf{k}^{\prime} ; \mathbf{x}_{D_{i_{D}}}\right)-G_{0}\left(\mathbf{k}, \mathbf{k}^{\prime}\right)\right]+\Delta G\left(\mathbf{k}, \mathbf{k}^{\prime} ; \mathbf{x}_{D_{1}}, \ldots, \mathbf{x}_{D_{N_{D}}}\right),
$$

where the remaining terms

$$
\Delta G\left(\mathbf{k}, \mathbf{k}^{\prime} ; \mathbf{x}_{D_{1}}, \ldots, \mathbf{x}_{D_{N_{D}}}\right)=\frac{1}{2} \sum_{\substack{1 \leq i_{D}, j_{D} \leq N_{D} \\\left(i_{D} \neq j_{D}\right)}}\left[G\left(\mathbf{k}, \mathbf{k}^{\prime} ; \mathbf{x}_{D_{i_{D}}}, \mathbf{x}_{D_{j_{D}}}\right)-G\left(\mathbf{k}, \mathbf{k}^{\prime} ; \mathbf{x}_{D_{i_{D}}}\right)-G\left(\mathbf{k}, \mathbf{k}^{\prime} ; \mathbf{x}_{D_{j_{D}}}\right)+G_{0}\left(\mathbf{k}, \mathbf{k}^{\prime}\right)\right]+\cdots
$$

can be expressed by two-, three-,.. . , -defect susceptibilities.

The configurational average

$$
\langle\langle\cdots\rangle\rangle=\prod_{j=1}^{N_{D}}\left(\frac{1}{N} \sum_{i_{D_{j}}=1}^{N}\right) \cdots
$$

of $\Delta G$ is of order $n^{2}$ (see, e.g., Appendix B of Ref. 46). Consequently, the multidefect response function can be found up to order $n$ from the single-defect susceptibility by averaging the first two terms of Eq. (B.3a):

$$
\left\langle\left\langle G\left(\mathbf{k}, \mathbf{k}^{\prime} ;\left\{\mathbf{x}_{D_{i_{D}}}\right\}\right)\right\rangle\right\rangle=G_{0}\left(\mathbf{k}, \mathbf{k}^{\prime}\right)+n^{\prime} \int d^{d} \mathbf{x}_{D}\left[G\left(\mathbf{k}, \mathbf{k}^{\prime} ; \mathbf{x}_{D}\right)-G_{0}\left(\mathbf{k}, \mathbf{k}^{\prime}\right)\right]+\boldsymbol{O}\left(n^{\prime 2}\right),
$$

with $n^{\prime}=N_{D} / V=n / a_{0}^{d}$. At the end of this section, we shall use (B4a) for the explicit computation in a one-dimensional model.

Now we show how the average of (B3a) is related to the single-site approximation used in the bulk of this paper. Use of $(\mathrm{B} 2 \mathrm{a}),(\mathrm{B} 2 \mathrm{~b})$, and (B3a) yields, for the defect-averaged phonon response function,

$$
\left\langle\left\langle G\left(\mathbf{k}, \mathbf{k}^{\prime}\right)\right\rangle=\delta_{\mathbf{k k}^{\prime}}\left[\frac{1}{\omega^{2}-\omega_{\mathbf{k}}^{2}}+n\left[\sum_{\mathbf{q}} \frac{f_{\mathbf{q}}(\mathbf{k}) f_{\mathbf{q}}^{*}(\mathbf{k})}{\omega^{2}-\varepsilon_{\mathbf{q}}}-\frac{1}{\omega^{2}-\omega_{\mathbf{k}}^{2}}\right]+O\left(n^{2}\right)\right],\right.
$$

which is translationally invariant, of course. Using the completeness relation for the eigenfunctions $f_{\mathbf{q}}$ the $\mathbf{q}$ sum on the right-hand side of (B4b) can be rearranged into

$$
\sum_{\mathbf{q}} \frac{f_{\mathbf{q}}(\mathbf{k}) f_{\mathbf{q}}^{*}(\mathbf{k})}{\omega^{2}-\varepsilon_{\mathbf{q}}}=\sum_{\mathbf{q}}\left\langle f_{\mathbf{q}} \mid \mathbf{k}\right\rangle\left\langle\mathbf{k}\left|\frac{1}{\omega^{2}-H}\right| f_{\mathrm{q}}\right\rangle=\left\langle\mathbf{k}\left|\frac{1}{\omega^{2}-H}\right| \mathbf{k}\right\rangle .
$$

Thus we find 


$$
\begin{aligned}
\left\langle\left\langle G\left(\mathbf{k}, \mathbf{k}^{\prime}\right)\right\rangle\right\rangle & =\delta_{\mathbf{k k}^{\prime}}\left[\frac{1}{\omega^{2}-\omega_{\mathbf{k}}^{2}}+n\left\langle\mathbf{k}|| \frac{1}{\omega^{2}-H}-\frac{1}{\omega^{2}-H_{0}}\right]|\mathbf{k}\rangle+O\left(n^{2}\right)\right] \\
& =\delta_{\mathbf{k k}^{\prime}}\left[\frac{1}{\omega^{2}-\omega_{\mathbf{k}}^{2}}+n\left\langle\mathbf{k}\left|\left[\frac{1}{\omega^{2}-H_{0}} V \frac{1}{\omega^{2}-H_{0}}+\frac{1}{\omega^{2}-H_{0}} V \frac{1}{\omega^{2}-H_{0}} V \frac{1}{\omega^{2}-H_{0}}+\cdots\right]\right| \mathbf{k}\right\rangle+O\left(n^{2}\right)\right],
\end{aligned}
$$

where in the second line we have expanded the resolvent $\left(\omega^{2}-H\right)^{-1}$ into a Born series.

If the defect potential is separable in momentum space,

$$
\left\langle\mathbf{k}|V| \mathbf{k}^{\prime}\right\rangle=\lambda \rho(\mathbf{k}) \rho^{*}\left(\mathbf{k}^{\prime}\right),
$$

the Born series (B4") can be summed explicitly by using the geometric sum formula

$$
\begin{aligned}
\left\langle\left\langle G\left(\mathbf{k}, \mathbf{k}^{\prime}\right)\right\rangle\right\rangle & =\delta_{\mathbf{k k}^{\prime}}\left[\frac{1}{\omega^{2}-\omega_{\mathbf{k}}^{2}}+\frac{n \lambda|\rho(\mathbf{k})|^{2}}{\left(\omega^{2}-\omega_{\mathbf{k}}^{2}\right)^{2}}\left[1+\frac{\lambda}{N} \sum_{\mathbf{q}} \frac{|\rho(\mathbf{q})|^{2}}{\omega^{2}-\omega_{\mathbf{q}}^{2}}+\cdots\right]+O\left(n^{2}\right)\right] \\
& =\delta_{\mathbf{k k}^{\prime}}\left(\frac{1}{\omega^{2}-\omega_{\mathbf{k}}^{2}}+\frac{n \lambda|\rho(\mathbf{k})|^{2}}{\left(\omega^{2}-\omega_{\mathbf{k}}^{2}\right)^{2}} \frac{1}{1-(\lambda / N) \sum_{\mathbf{q}}|\rho(\mathbf{q})|^{2} /\left(\omega^{2}-\omega_{\mathbf{q}}^{2}\right)}+O\left(n^{2}\right)\right],
\end{aligned}
$$

which exactly corresponds to the summation in (4.17). At last, Eq. (B7) is readily interpreted as the first-order term of the expansion of a Dyson equation. Thus we find the final result:

$$
\left\langle\left\langle G\left(\mathbf{k}, \mathbf{k}^{\prime}\right)\right\rangle\right\rangle=\delta_{\mathbf{k}, \mathbf{k}^{\prime}} /\left(\omega^{2}-\omega_{\mathbf{k}}^{2}-\frac{n \lambda|\rho(\mathbf{k})|^{2}}{1-\frac{\lambda}{N} \sum_{\mathbf{q}}|\rho(\mathbf{q})|^{2} /\left(\omega^{2}-\omega_{\mathbf{q}}^{2}\right)}\right),
$$

which in the special case $\rho(\mathbf{k})=1$, applying to the local defect potential used in Sec. IV and after identifying $\omega^{2}-\omega_{\mathbf{k}}^{2}$ with $\left[G_{0}(\mathbf{k}, \omega)\right]^{-1}$, is of the form (4.16).

Now we rederive Eqs. (4.16) and (4.17) for the one-dimensional model, i.e., with the special form of the self-energy given by Eq. $(4.24 \mathrm{c})$, where the single-defect correlation function can be explicitly computed. Introducing dimensionless quantities for convenience according to

$$
\begin{aligned}
& z=\left(\frac{|a|}{c}\right)^{1 / 2} x, \quad k=\left(\frac{c}{|a|}\right)^{1 / 2} q, \quad c^{\prime}=\left(\frac{c}{|a|}\right)^{1 / 2} n^{\prime}, \\
& v=\left(\frac{I}{|a|}\right)^{1 / 2} \omega, \quad D=\left(\frac{I}{|a|}\right)^{1 / 2} \gamma, \quad v_{0}=\frac{U}{\sqrt{|a| c}},
\end{aligned}
$$

the equation of motion for the single-defect system (3.10) in one dimension and for $T>T_{c}^{l}$ obtains the simple form

$$
v_{\lambda}^{2} u_{\lambda}(z)=\left(1-v_{0} \delta(z)-\frac{d^{2}}{d z^{2}}\right) u_{\lambda}(z)
$$

Its solutions read

$$
\begin{aligned}
& u_{k}^{+}(z)=\frac{k \cos (k z)-\kappa \sin (k|z|)}{\left[\pi\left(k^{2}+\kappa^{2}\right)\right]^{1 / 2}}, \\
& u_{k}^{-}(z)=\frac{\sin (k z)}{\sqrt{\pi}}, \\
& u_{0}(z)=\sqrt{\kappa} e^{-\kappa|z|}, \quad \kappa=\frac{v_{0}}{2} .
\end{aligned}
$$

The scattering states (B11a) and (B11b) belonging to the eigenvalue $v_{k}^{2}=1+k^{2}$ fulfill the following orthogonality relations:

$$
\int_{-\infty}^{+\infty} u_{k}^{ \pm}(z) u_{k^{\prime}}^{ \pm}(z) d z=\delta\left(k-k^{\prime}\right)
$$

while the bound state (B11c) with $v_{0}^{2}=1-\kappa^{2}$ is normalized according to

$$
\int_{-\infty}^{+\infty}\left[u_{0}(z)\right]^{2} d z=1
$$

One can easily confirm the completeness relation 


$$
\int_{0}^{\infty}\left[u_{k}^{+}(z) u_{k}^{+}\left(z^{\prime}\right)+u_{k}^{-}(z) u_{k}^{-}\left(z^{\prime}\right)\right] d k+u_{0}(z) u_{0}\left(z^{\prime}\right)=\delta\left(z-z^{\prime}\right)
$$

The one-defect response function can now be exactly represented by a summation over the normalized eigenfunctions divided by an "energy denominator" [see Eq. (B2a)]:

$$
G\left(z, z^{\prime} ; 0\right)=\int_{0}^{\infty} \frac{u_{k}^{+}(z) u_{k}^{+}\left(z^{\prime}\right)+u_{k}^{-}(z) u_{k}^{-}\left(z^{\prime}\right)}{v_{k}^{2}-v(v+i D)} d k+\frac{u_{0}(z) u_{0}\left(z^{\prime}\right)}{1-k^{2}-v(v+i D)} .
$$

Now, placing the defect at position $z_{D}$, the integration can be readily performed:

$$
G\left(z, z^{\prime} ; z_{D}\right)=\frac{1}{2 \mu} e^{-\mu\left|z-z^{\prime}\right|}-\frac{\kappa}{2 \mu(\kappa-\mu)} e^{-\mu\left(\left|z-z_{D}\right|+\left|z^{\prime}-z_{D}\right|\right)},
$$

with the abbreviation $\mu=\sqrt{1-v(v+i D)}$. Of course, the one-defect phonon response function is not translationally invariant. Only the first term, which is identical to the phonon response function of a pure system,

$$
G_{0}\left(z-z^{\prime}\right)=\frac{1}{2 \mu} e^{-\mu\left|z-z^{\prime}\right|},
$$

is translationally invariant.

Using now Eq. (B4a), we obtain, for the defect-averaged response function,

$$
\left\langle\left\langle G\left(z-z^{\prime}\right)\right\rangle\right\rangle=\frac{1}{2 \mu} e^{-\mu\left|z-z^{\prime}\right|}\left[1-\frac{c^{\prime} \kappa}{2 \mu(\kappa-\mu)}\left[\frac{1}{\mu}+\left|z-z^{\prime}\right|\right]+O\left(c^{\prime 2}\right)\right],
$$

which is translationally invariant. Its Fourier transform reads

$$
\langle\langle G(k)\rangle\rangle=\frac{1}{\mu^{2}+k^{2}}-\frac{2 c^{\prime} \kappa \mu}{(\kappa-\mu)\left(\mu^{2}+k^{2}\right)^{2}}+O\left(c^{\prime 2}\right) .
$$

If again we interpret this as the first-order expansion of a self-energy correction, we end up with

$$
\langle\langle G(k)\rangle\rangle=\frac{1}{\mu^{2}+k^{2}-2 c^{\prime} \kappa \mu /(\mu-\kappa)} .
$$

Inserting the definitions of $\kappa, \mu$ and rescaling to the original variables according to Eq. (B9) shows that the result (B18) is identical to those of Sec. IV obtained for the one-dimensional case in single-site approximation. We remark that in the case of elastic phase transitions this procedure has already been employed. ${ }^{45}$

${ }^{1}$ R. Brout, Phys. Rev. 115, 824 (1959).

${ }^{2}$ M. E. Fisher, Phys. Rev. 176, 257 (1968).

${ }^{3}$ T. Riste, E. J. Samuelsen, K. Otnes, and J. Feder, Solid State Commun. 9, 1455 (1971).

${ }^{4}$ S. M. Shapiro, J. D. Axe, G. Shirane, and T. Riste, Phys. Rev. B 6, 4332 (1972).

${ }^{5}$ J. K. Kjems, G. Shirane, K. A. Müller, and H. J. Scheel, Phys. Rev. B 8, 1119 (1973).

${ }^{6}$ F. Denoyer, M. Lambert, A. Comes, and R. Currat, Solid State Commun. 18, 441 (1976).

${ }^{7}$ R. A. Cowley, J. D. Axe, and M. Iizumi, Phys. Rev. Lett. 36, 806 (1976).

${ }^{8}$ P. A. Fleury and K. B. Lyons, Phys. Rev. Lett. 37, 1088 (1976).

${ }^{9}$ E. Courtens, Phys. Rev. Lett. 41, 1171 (1978).

${ }^{10}$ T. Yagi, H. Tanaka, and I. Tatzuki, Phys. Rev. Lett. 38, 609 (1977).

${ }^{11}$ K. A. Müller, in Dynamical Critical Phenomena and Related Topics, edited by C. P. Enz (Springer-Verlag, Berlin, 1979), p. 210.

${ }^{12}$ J. Töpler, B. Alefeld, and A. Heidemann, J. Phys. C 10, 635 (1977).

${ }^{13} \mathrm{~F}$. Mezei and J. Hayter (unpublished).

${ }^{14}$ C. N. W. Darlington and D. A. O'Connor, J. Phys. C 9, 3561 (1976).
${ }^{15}$ K. Hanisch and M. Drosg, Phys. Lett. 58A, 415 (1976).

${ }^{16}$ K. B. Lyons and P. A. Fleury, Phys. Rev. B 17, 2403 (1976); Phys. Rev. Lett. 37, 161 (1976).

${ }^{17}$ L. N. Durvasula and R. W. Gammon, Phys. Rev. Lett. 38, 1081 (1977).

${ }^{18}$ J. B. Hastings, S. M. Shapiro, and B. C. Frazer, Phys. Rev. Lett. 40, 237 (1978).

${ }^{19}$ D. Wagner, D. Bäuerle, F. Schwabl, B. Dorner, and H. Kraxenberger, Z. Phys. B 37, 317 (1980).

${ }^{20}$ F. Schwabl, Z. Phys. 254, 57 (1972); Phys. Rev. Lett. 28, 500 (1972).

${ }^{21}$ R. A. Cowley, J. Phys. Soc. Jpn. Suppl. 28, 239 (1974).

${ }^{22}$ R. Silberglitt, Solid State Commun. 11, 247 (1972).

${ }^{23}$ C. P. Enz, Phys. Rev. B 6, 4695 (1972).

${ }^{24}$ F. Schwabl, Solid State Commun. 13, 181 (1973); Ferroelectrics 7, 395 (1974).

${ }^{25}$ K. K. Murata, Phys. Rev. B 11, 462 (1975).

${ }^{26}$ J. Axe, S. M. Shapiro, G. Shirane, and T. Riste, in Anharmonic Lattices, Structural Transitions and Melting, edited by T. Riste (Noordhoff, Leiden, 1974), p. 23.

${ }^{27}$ F. Schwabl, in Ref. 26, p. 87.

${ }^{28}$ R. Folk and F. Schwabl, Solid State Commun. 15, 937 (1974).

${ }^{29}$ F. Schwabl, in Ferroelektrische Phasenübergänge, 3. Frühjahrsschule "Ferroelektrizität," edited by W. Windsch (Martin-Luther-Universität, Halle, 1975), p. 47. 
${ }^{30}$ B. I. Halperin and C. M. Varma, Phys. Rev. B 14, 4030 (1976).

${ }^{31}$ H. Schmidt and F. Schwabl, Phys. Lett. 61A, 476 (1977); in Proceedings of the International Conference on Lattice Dynamics, edited by M. Balkanski (Flammarion Sciences, Paris, 1977), p. 748.

${ }^{32}$ H. Schmidt and F. Schwabl, Z. Phys. B 30, 197 (1978).

${ }^{33}$ K. H. Höck and H. Thomas, Z. Phys. B 27, 267 (1977).

${ }^{34}$ K. H. Höck, R. Schäfer, and H. Thomas, Z. Phys. B 36, 151 (1979).

${ }^{35}$ K. H. Weyrich and R. Siems, Ferroelectrics 55, 333 (1984).

${ }^{36}$ B. Wiesen, K. H. Weyrich, and R. Siems, Jpn. J. Appl. Phys. 24, Suppl. 24-2, 995 (1985); Phys. Rev. B 36, 3175 (1987); Ferroelectrics 79, 69 (1988).

${ }^{37}$ N. Kristoffel and M. Klopov, Phys. Status Solidi B 151, K119 (1989); in Phonons '89, Proceedings of the 3rd International Conference on Phonon Physics and of the 6th International
Conference on Phonon Scattering in Condensed Matter, edited by S. Hunklinger, W. Ludwig, and G. Weiss (World Scientific, Singapore, 1990), Vol. 2, p. 1208.

${ }^{38}$ R. Pöschko, Diplomarbeit Universität Linz, 1980.

${ }^{39}$ R. G. Petschek, Phys. Rev. B 22, 1409 (1980).

${ }^{40}$ J. S. Langer, J. Math. Phys. 2, 584 (1961).

${ }^{41}$ R. J. Elliott, J. A. Krumhansl, and P. L. Leath, Rev. Mod. Phys. 46, 465 (1974).

${ }^{42}$ D. J. Amit, Field Theory, the Renormalization Group, and Critical Phenomena (World Scientific, Singapore, 1984).

${ }^{43}$ G. F. Mazenko, Phys. Rev. B 14, 3933 (1976).

${ }^{44}$ L. Sasvári, F. Schwabl, and P. Szépfalusy, Physica A 81, 108 (1975).

${ }^{45}$ F. Schwabl and U. C. Täuber, in Ref. 37, Vol. 2, p. 1138; Phase Transitions (to be published).

${ }^{46}$ H. Reisinger and F. Schwabl, Z. Phys. B 52, 151 (1983). 